\title{
HARD LEFSCHETZ PROPERTY FOR HAMILTONIAN TORUS ACTIONS ON 6-DIMENSIONAL GKM MANIFOLDS
}

\author{
YUNHYUNG CHO AND MIN KYU KIM
}

\begin{abstract}
Let $(M, \omega)$ be a 6 -dimensional closed symplectic manifold with a Hamiltonian $T^{2}$-action. We show that if the action is GKM and its GKM graph is index-increasing, then $(M, \omega)$ satisfies the hard Lefschetz property.
\end{abstract}

\section{Contents}

1. Introduction 1

2. Equivariant cohomology 3

3. The Graph cohomology of Hamiltonian GKM manifolds 6

4. Hodge-Riemann bilinear forms 9

5. Six-dimensional Hamiltonian GKM manifolds with index increasing graphs 13

6. Proof of Proposition 5.15 and 5.17 20

\begin{tabular}{l} 
References \\
\hline
\end{tabular}

\section{InTRODUCTION}

Let $T$ be a compact torus acting effectively on a closed symplectic manifold $(M, \omega)$ in a Hamiltonian fashion. If the T-action is GKM, the celebrated theorem [GKM, Theorem 1.2.2] due to GoreskyKottwitz-MacPherson tells us that the equivariant cohomology $\operatorname{ring}$ of $M$ is completely determined by the corresponding GKM graph, which is an image of zero and one-dimensional torus orbits in $M$ under a moment map. In particular, since the ordinary cohomology $\operatorname{ring} H^{*}(M ; \mathbb{R})$ of $M$ can be obtained from its equivariant cohomology ring by extension of scalars, the product structure of $H^{*}(M ; \mathbb{R})$ is determined by the GKM graph.

In this paper, we study the hard Lefschetz property of a closed Hamiltonian GKM manifold. We say that a closed symplectic manifold $(M, \omega)$ satisfies the hard Lefschetz property if

$$
\begin{aligned}
\wedge[\omega]^{n-l}: H^{l}(M ; \mathbb{R}) & \longrightarrow H^{2 n-l}(M ; \mathbb{R}) \\
\alpha & \longmapsto \alpha \wedge[\omega]^{n-l}
\end{aligned}
$$

is an isomorphism for every $l=0,1, \cdots, n$. It is clear that the product structure of $H^{*}(M ; \mathbb{R})$ and the cohomology class $[\omega] \in H^{2}(M ; \mathbb{R})$ determine whether $(M, \omega)$ satisfies the hard Lefschetz property or not, and therefore it is natural to ask how to check the hard Lefschetz property of a closed Hamiltonian GKM manifold by "looking up" the corresponding GKM graph.

It is known that the hard Lefschetz property does not hold in general. See [Cho1] or [Go] for example. However, it is not known whether $(M, \omega)$ satisfies the hard Lefschetz property when $(M, \omega)$ admits a Hamiltonian torus action with isolated fixed points. Our work is motivated by the following question posed by Karshon.

Question 1.1. [JHKLM] Let $(M, \omega)$ be a closed symplectic manifold with an effective Hamiltonian circle action. Assume that all fixed points are isolated. Then, does $(M, \omega)$ satisfy the hard Lefschetz property? 
Note that if $(M, \omega)$ satisfies the hard Lefschetz property, then we can easily see that the sequence $\left\{b_{0}(M), b_{2}(M), \cdots, b_{2 n}(M)\right\}$ is unimodal ${ }^{1}$ where $b_{i}(M)$ denotes the $i$-th Betti number of $M$. This leads to the following question, posed by Tolman, regarded as a weak version of Question 1.1.

Question 1.2. [JHKLM] Let $(M, \omega)$ be a closed symplectic manifold with an effective Hamiltonian circle action. If all fixed points are isolated, then is the sequence $\left\{b_{0}(M), b_{2}(M), \cdots, b_{2 n}(M)\right\}$ unimodal?

Following a remark by Karshon in [JHKLM], we observe that the condition of "admitting isolated fixed points" is a strong assumption in the sense that an example of a closed symplectic non-Kähler Hamiltonian $S^{1}$-manifold with isolated fixed points has not been found so far. In fact, there are several positive results on Question 1.1 and Question 1.2 for a Hamiltonian torus action with isolated fixed points. For example, Delzant [De] proved that every closed symplectic toric manifold is Kähler and hence the hard Lefschetz property holds. Also, Karshon [Ka] proved that any four dimensional closed Hamiltonian $S^{1}$-manifold $(M, \omega)$ with isolated fixed points admits an $S^{1}$-invariant Kähler form. In this case, the hard Lefschetz property is rather obvious since $H^{1}(M ; \mathbb{R})=H^{3}(M ; \mathbb{R})=0$ by the Frankel's theorem [Fr, Corollary 2]. Also, some positive answers to Question 1.1 and Question 1.2 are provided in [Cho2], [CK1], [CK2], and [Lu] under certain technical assumptions.

Throughout this paper we restrict our attention to Question 1.1 for closed Hamiltonian GKM manifolds. Note that $(M, \omega)$ satisfies the hard Lefschetz property if and only if the Hodge-Riemann bilinear form defined as

$$
\begin{aligned}
& \mathrm{HR}_{l}: H^{l}(M) \times H^{l}(M) \quad \longrightarrow \quad \mathbb{R} \\
& (\alpha, \beta) \quad \longmapsto<\alpha \beta[\omega]^{n-l},[M]>
\end{aligned}
$$

is non-degenerate for every $l=0,1, \cdots, n$. To check the non-degeneracy of $\mathrm{HR}_{l}$, we first consider certain two bases $\mathcal{B}_{l}^{+}$and $\mathcal{B}_{l}^{-}$of $H^{l}(M ; \mathbb{R})$, which consist of so-called the equivariant Thom classes in $H_{T}^{l}(M ; \mathbb{R})$ introduced by Guillemin-Zara [GZ]. (See also Section 3.) Then we show that the matrix, denoted by $A_{l}(M, \omega)$, representing $\mathrm{HR}_{l}$ with respect to the pair $\left(\mathcal{B}_{l}^{+}, \mathcal{B}_{l}^{-}\right)$is obtained from the GKM graph by using the ABBV-localization theorem and Goldin-Tolman's theorem [GT]. (See Proposition 4.4 for the detail.) Also, in case of $n-l=1$, we show that $A_{l}(M, \omega)$ has many zero entries. (See Corollary 4.7.) Furthermore, we prove the following if $M$ is of dimension six.

Theorem 1.3. Let $(M, \omega)$ be a 6 -dimensional closed symplectic manifold equipped with an effective Hamiltonian $T^{2}$-action. If the action is GKM and the corresponding GKM graph is index increasing ${ }^{2}$, then $(M, \omega)$ satisfies the hard Lefschetz property.

Example 1.4. In [T], Tolman constructed a six-dimensional closed Hamiltonian GKM manifold $(M, \omega)$ which has no Kähler metric invariant under the action. The corresponding GKM graph is given in Figure 1.1 .

With respect to the $\xi$ described in Figure 1.1, we see that the GKM graph is index-increasing so that Tolman's manifold satisfies the hard Lefschetz property by Theorem 1.3. In fact, Woodward already pointed out in [Wo2, page 9] that Tolman's manifold satisfies the hard Lefschetz property with a hint for a proof, which seems to rely on computation of the cohomology ring of $M$. Also, he constructed more examples of non-Kähler GKM-manifolds using $U(2)$-equivariant surgery and they have the same x-ray with Tolman's example [Wo, Proposition 3.6], and therefore their GKM graphs are all index-increasing. Consequently, every Woodward's example satisfies the hard Lefschetz property by Theorem 1.3.

Organization. In Section 2, we give a brief introduction to the equivariant cohomology theory for Hamiltonian torus actions and recall the ABBV-localization theorem which will be used in order to compute the matrix $A_{l}(M, \omega)$ representing the Hodge-Riemann bilinear form $\mathrm{HR}_{l}$. In Section 3, we

\footnotetext{
${ }^{1} \mathrm{~A}$ sequence of real numbers $a_{1}, \cdots, a_{n}$ is called unimodal if there exists an integer $k \geq 1$ such that $a_{1} \leq \cdots \leq a_{k} \geq$ $\cdots \geq a_{n}$

${ }^{2}$ See Definition 3.3 .
} 


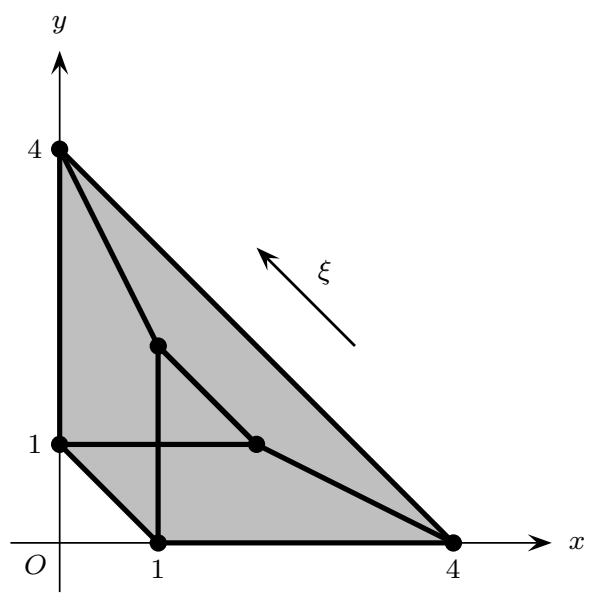

FIGURE 1.1. Tolman's Hamiltonian GKM manifold

provide some background on Hamiltonian GKM manifolds and their graph cohomology rings. In Section 4, we compute the matrix $A_{l}(M, \omega)$ by using combinatorial data of a GKM graph. In Section 5, we prove our main theorem (Theorem 1.3). Finally, in Section 6, we prove two propositions crucially used in Section 5 .

Acknowledgement. The authors thank anonymous referees for their endurance and kindness to improve the paper, especially to bring the beautiful papers [GT], [ST], and [Mo] to our attention. The first author was supported by the National Research Foundation of Korea(NRF) grant funded by the Korea government(MSIP; Ministry of Science, ICT \& Future Planning) (NRF-2017R1C1B5018168). The second author is supported by GINUE research fund.

\section{EquiVARIANT COHOMOLOGY}

Throughout this paper, we assume that an action of a Lie group on a manifold is effective, unless stated otherwise. Also, we take cohomology with coefficients in $\mathbb{R}$.

Let $(M, \omega)$ be a closed symplectic manifold admitting Hamiltonian $T$-action where $T$ is a compact $m$-dimensional torus for some integer $m \geq 1$. Then the equivariant cohomology of $M$ is defined by

$$
H_{T}^{*}(M)=: H^{*}\left(M \times_{T} E T\right)
$$

where $E T$ is a contractible space on which $T$ acts freely. In particular, the equivariant cohomology of a point is given by $H_{T}^{*}(\mathrm{pt})=H^{*}\left(\mathrm{pt} \times_{T} E T\right)=H^{*}(B T)$ where $B T=E T / T$ is the classifying space of $T$. Note that if $T=S^{1}$, then $B S^{1}$ can be constructed as an inductive limit of the sequence of Hopf fibrations

$$
\begin{array}{ccccccccc}
S^{3} & \hookrightarrow & S^{5} & \hookrightarrow & \cdots & S^{2 n+1} & \ldots & \hookrightarrow & E S^{1} \sim S^{\infty} \\
\downarrow & & \downarrow & & \cdots & \downarrow & \ldots & & \downarrow \\
\mathbb{C} P^{1} & \hookrightarrow & \mathbb{C} P^{2} & \hookrightarrow & \cdots & \mathbb{C} P^{n} & \ldots & \hookrightarrow & B S^{1} \sim \mathbb{C} P^{\infty}
\end{array}
$$

Thus we have

$$
H^{*}\left(B S^{1}\right) \cong \mathbb{R}[x]
$$

where $x$ is an element of degree two such that $\left\langle x,\left[\mathbb{C} P^{1}\right]\right\rangle=1$. Similarly, if we choose an ordered $\mathbb{Z}$-basis $\mathfrak{X}=\left\{X_{1}, \cdots, X_{m}\right\}$ for the lattice ${ }^{3}$ in $\mathfrak{t}$ and a decomposition $T=S^{1} \times \cdots \times S^{1}$ corresponding to $\mathfrak{X}$, then we can easily check that $B T$ is homotopy equivalent to the $m$-times product of $\mathbb{C} P^{\infty}$ and hence

$$
H^{*}(B T) \cong \mathrm{S}\left(\mathfrak{t}^{*}\right)=\mathbb{R}\left[x_{1}, \cdots, x_{m}\right]
$$

\footnotetext{
${ }^{3}$ The lattice of $\mathfrak{t}$ means the kernel of the exponential map from $\mathfrak{t}$ to $T$.
} 
where $\mathrm{S}\left(\mathfrak{t}^{*}\right)$ is the symmetric tensor algebra of $\mathfrak{t}^{*}$ and each $x_{i} \in \mathfrak{t}^{*}$ is the dual of $X_{i}$ and is of degree two for $i=1, \cdots, m$.

2.1. Equivariant formality. Note that a projection map $M \times E T \rightarrow E T$ on the second factor is $T$ equivariant so that it induces the map

$$
\pi: M \times_{T} E T \rightarrow B T
$$

which makes $M \times_{T} E T$ into an $M$-bundle over $B T$

$$
\begin{aligned}
& M \times_{T} E T \stackrel{f}{\hookleftarrow} M \\
& \pi \downarrow \\
& B T
\end{aligned}
$$

where $f$ is an inclusion of a fiber $M$. Then it induces the following sequence

$$
H^{*}(B T) \stackrel{\pi^{*}}{\rightarrow} H_{T}^{*}(M) \stackrel{f^{*}}{\rightarrow} H^{*}(M) .
$$

In particular, $H_{T}^{*}(M)$ has an $H^{*}(B T)$-module structure via the map $\pi^{*}$ such that

$$
x \cdot \alpha=\pi^{*}(x) \cup \alpha
$$

for $x \in H^{*}(B T)$ and $\alpha \in H_{T}^{*}(M)$.

Definition 2.1. Let $(M, \omega)$ be a symplectic manifold. We say that a $T$-action on $(M, \omega)$ is Hamiltonian if there exists a smooth map $\mu: M \rightarrow \mathfrak{t}^{*}$ such that

$$
d\langle\mu, X\rangle=\omega(X, \cdot)
$$

for every $X \in \mathfrak{t}$. We call $\mu$ a moment map for the $T$-action.

Remark 2.2. Note that if $\mu$ is a moment map for a Hamiltonian $T$-action on $(M, \omega)$, then $\mu+c$ is also a moment map for any $c \in \mathfrak{t}^{*}$. Thus a moment map is not unique.

The equivariant cohomology of Hamiltonian $T$-action has a remarkable property as follows.

Theorem 2.3. [Ki] Let $(M, \omega)$ be a closed symplectic manifold equipped with a Hamiltonian T-action. Then $M$ is equivariantly formal, that is, $H_{T}^{*}(M)$ is a free $H^{*}(B T)$-module so that

$$
H_{T}^{*}(M) \cong H^{*}(M) \otimes H^{*}(B T) .
$$

Equivalently, the map $f^{*}$ is surjective with the kernel $\left\langle x_{1}, \cdots, x_{m}\right\rangle \cdot H_{T}^{*}(M)$ where $\left\langle x_{1}, \cdots, x_{m}\right\rangle$ is an ideal of $H^{*}(B T)$ generated by degree two elements $x_{1}, \cdots, x_{m}$ and $\cdot$ denotes the scalar multiplication of the $H^{*}(B T)$-module structure on $H_{T}^{*}(M)$.

2.2. Localization theorem. For a given $k \in \mathbb{Z}_{\geq 0}$ and an element $\alpha \in H_{T}^{k}(M)$, Theorem 2.3 implies that $\alpha$ can be uniquely expressed as

$$
\alpha=\alpha_{k} \otimes 1+\sum_{i=1}^{m} \alpha_{k-2}^{i} \otimes x_{i}+\sum_{1 \leq i, j \leq m} \alpha_{k-4}^{i, j} \otimes x_{i} x_{j}+\cdots
$$

where $\alpha_{i}^{J} \in H^{i}(M)$ for every $i \leq k$ and $J$ is a multiset whose elements are in $[m]=\{1, \cdots, m\}$. We denote the set of multisets with elements in $[m]$ by $[m]^{\text {mul }}$. With this notation, we have $f^{*}(\alpha)=\alpha_{k}$.

Definition 2.4. An integration along the fiber $M$ is an $H^{*}(B T)$-module homomorphism $\int_{M}: H_{T}^{*}(M) \rightarrow$ $H^{*}(B T)$ defined by

$$
\int_{M} \alpha=\left\langle\alpha_{k},[M]\right\rangle \cdot 1+\sum_{i=1}^{m}\left\langle\alpha_{k-2}^{i},[M]\right\rangle \cdot x_{i}+\cdots
$$

for every $k \in \mathbb{Z}_{\geq 0}$ and any $\alpha \in H_{T}^{k}(M)$ where $[M]$ is the fundamental homology class of $M$. 
Note that $\left\langle\alpha_{j}^{J},[M]\right\rangle=0$ for any $J \subset[m]^{\text {mul }}$ and $j<\operatorname{dim} M=2 n$. Also, $\alpha_{j}^{J}=0$ for every $j>2 n$ for a dimensional reason, and therefore we have

$$
\int_{M} \alpha=\sum_{\substack{J \in[m]^{\mathrm{mul}} \\|J|+2 n=k}}\left\langle\alpha_{2 n}^{J},[M]\right\rangle x^{J}
$$

for every $k \in \mathbb{Z}_{\geq 0}$ and any $\alpha \in H_{T}^{k}(M)$ where $x^{J}=\prod_{j \in J} x_{j}$. This leads to the following corollary.

Corollary 2.5. Let $\alpha \in H_{S^{1}}^{*}(M)$ such that $\operatorname{deg} \alpha \leq \operatorname{dim} M$. Then we have

$$
\int_{M} \alpha=\left\langle f^{*}(\alpha),[M]\right\rangle \text {. }
$$

Let $M^{T}$ be the fixed point set and let $F \subset M^{T}$ be a fixed component with an inclusion map $i_{F}: F \hookrightarrow M$. Then it induces a ring homomorphism

$$
i_{F}^{*}: H_{T}^{*}(M) \rightarrow H_{T}^{*}(F) \cong H^{*}(F) \otimes H^{*}(B T) .
$$

For any $\alpha \in H_{T}^{*}(M)$, the image $i_{F}^{*}(\alpha)$ is called the restriction of $\alpha$ to $F$ and is denoted by $\left.\alpha\right|_{F}$. The following theorem due to Atiyah-Bott [AB] and Berline-Vergne [BV] states that the integration $\int_{M} \alpha$ can be calculated in terms of the fixed point data.

Theorem 2.6. ( $A B B V$-localization) For any $\alpha \in H_{T}^{*}(M)$, we have

$$
\int_{M} \alpha=\sum_{F \subset M^{T}} \int_{F} \frac{\left.\alpha\right|_{F}}{\Lambda_{F}}
$$

where $\Lambda_{F}$ is the equivariant Euler class of the normal bundle of $F$. In particular, if every fixed point is isolated, then

$$
\int_{M} \alpha=\sum_{F \in M^{T}} \frac{\left.\alpha\right|_{F}}{\Lambda_{F}}
$$

Recall that the $l$-th Hodge-Riemann bilinear form is given by

$$
\begin{aligned}
& \mathrm{HR}_{l}: H^{l}(M) \times H^{l}(M) \longrightarrow \mathbb{R} \\
& (\alpha, \beta) \quad \longmapsto<\alpha \beta[\omega]^{n-l},[M]>
\end{aligned}
$$

for $l=0,1, \cdots, n$. Let $\alpha$ and $\beta$ be any elements in $H^{l}(M)$. Since $f^{*}$ is surjective by Theorem 2.3, we can find $\widetilde{\alpha}, \widetilde{\beta},[\widetilde{\omega}] \in H_{T}^{*}(M)$ such that $f^{*}(\widetilde{\alpha})=\alpha, f^{*}(\widetilde{\beta})=\beta$, and $f^{*}([\widetilde{\omega}])=[\omega]$ and hence we get

$$
\int_{M} \widetilde{\alpha} \widetilde{\beta}[\widetilde{\omega}]^{n-l}=\left\langle\alpha \beta[\omega]^{n-l},[M]\right\rangle
$$

by Corollary 2.5. Thus we can compute $\left\langle\alpha \beta[\omega]^{n-l},[M]\right\rangle$ by applying the ABBV-localization theorem to $\widetilde{\alpha} \widetilde{\beta}[\widetilde{\omega}]^{n-l}$.

2.3. Cartan models. Note that the choice of a class $[\widetilde{\omega}] \in H_{T}^{2}(M)$ satisfying $f^{*}([\widetilde{\omega}])=[\omega]$ is parametrized by a moment map. To understand $[\widetilde{\omega}]$ in more detail, we briefly overview the Cartan model of $H_{T}^{*}(M)$ as follows. (See also [GS2].) Let us consider the set of equivariant q-forms

$$
\Omega_{T}^{q}(M)=\bigoplus_{2 i+j=q} S^{i}\left(\mathfrak{t}^{*}\right) \otimes \Omega^{j}(M)^{T}
$$

where $S^{i}\left(\mathfrak{t}^{*}\right)$ denotes the set of degree $i$ elements in the symmetric tensor algebra of $\mathfrak{t}^{*}$ and $\Omega^{j}(M)^{T}$ is the set of $T$-invariant differential $j$-forms on $M$. Then we may think of an element $\alpha \in \Omega_{T}^{*}(M)$ as a map from $\mathfrak{t}$ to $\Omega^{*}(M)^{T}$. We call $\left(\Omega_{T}^{*}, d_{T}\right)$ the Cartan complex where the differential is defined by

$$
d_{T}:=1 \otimes d+\sum_{j=1}^{m} x_{i} \otimes i_{X_{i}}, \quad d_{T}(f \otimes \alpha)=f \otimes d \alpha+\sum_{j=1}^{m} x_{i} f \otimes i_{X_{i}} \alpha
$$


for any $f \otimes \alpha \in S^{*}\left(\mathfrak{t}^{*}\right) \otimes \Omega^{*}(M)^{T}$ where $\left\{X_{1}, \cdots, X_{m}\right\}$ and $\left\{x_{1}, \cdots, x_{m}\right\}$ are the basis, which we have chosen in (2.1), of $\mathfrak{t}$ and $\mathfrak{t}^{*}$, respectively. Then it is not hard to check that $d_{T}^{2}=0$ by direct computation. The equivariant de Rham theorem states that

$$
H_{T}^{*}(M) \cong H\left(\Omega_{T}^{*}(M), d_{T}\right) .
$$

Now, let $\mu=\left(\mu_{1}, \cdots, \mu_{m}\right): M \rightarrow \mathfrak{t}^{*}$ be a moment map where $m=\operatorname{dim} T$. Since each component of $\mu$ is $T$-invariant, we may regard $\mu$ as an element of $S^{1}\left(\mathfrak{t}^{*}\right) \otimes \Omega^{0}(M)^{T} \subset \Omega_{T}^{2}(M)$ such that

$$
\mu=x_{1} \otimes \mu_{1}+\cdots+x_{m} \otimes \mu_{m} .
$$

Since $\omega$ is also $T$-invariant, $\omega$ can be regarded as the element $1 \otimes \omega \in S^{0}\left(\mathfrak{t}^{*}\right) \otimes \Omega^{2}(M)^{T} \subset \Omega_{T}^{2}(M)$. Define

$$
\widetilde{\omega}_{\mu}:=\omega-\mu=1 \otimes \omega-\sum_{i=1}^{m} x_{i} \otimes \mu_{i} \in \Omega_{T}^{2}(M) .
$$

We call $\widetilde{\omega}_{\mu}$ the equivariant symplectic form with respect to $\mu$. Then

$$
\begin{aligned}
d_{T}\left(\widetilde{\omega}_{\mu}\right) & =d_{T}(\omega-\mu) \\
& =1 \otimes d \omega-\sum_{j=1}^{m} x_{i} \otimes d \mu_{i}+\sum_{j=1}^{m} x_{i} \otimes i_{X_{i}} \omega \\
& =\sum_{j=1}^{m} x_{i} \otimes\left(i_{X_{i}} \omega-d \mu_{i}\right) \\
& =0
\end{aligned}
$$

so that $\widetilde{\omega}_{\mu}$ is $d_{T}$-closed, and therefore $\widetilde{\omega}_{\mu}$ represents an equivariant cohomology class $\left[\widetilde{\omega}_{\mu}\right] \in H_{T}^{2}(M)$ which we call the equivariant symplectic class with respect to $\mu$. Then we immediately obtain the following corollary from the definition of $\widetilde{\omega}_{\mu}$.

Lemma 2.7. Let $v \in M^{T}$ be an isolated fixed point. Then

$$
\left.\left[\widetilde{\omega}_{\mu}\right]\right|_{v}=-\sum_{j=1}^{m} x_{i} \otimes \mu_{i}(v)=-\mu(v) \in \mathfrak{t}^{*}=S^{1}\left(\mathfrak{t}^{*}\right) \cong H^{2}(B T) .
$$

\section{The Graph cohomology of Hamiltonian GKM manifolds}

In this section, we briefly review the theory of Hamiltonian GKM-manifolds and GKM graphs, following [GKM] and [GZ].

3.1. GKM manifolds. Let $(M, \omega)$ be a $2 n$-dimensional closed symplectic manifold and let $T$ be an $m$ dimensional torus with its Lie algebra $\mathfrak{t}$ for some integer $m \geq 2$. Suppose that $T$ acts on $(M, \omega)$ in a Hamiltonian fashion with a moment map $\mu: M \longrightarrow \mathfrak{t}^{*}$.

Definition 3.1. The triple $(M, \omega, \mu)$ is called a Hamiltonian GKM manifold if

(1) the fixed point set $M^{T}$ is finite, and

(2) for each $v \in M^{T}$, the weights $\alpha_{j, v} \in \mathfrak{t}^{*}, j=1, \cdots, n$, of the one-dimensional isotropy $T$ representations on $T_{v} M$ are pairwise linearly independent.

A Hamiltonian GKM manifold $(M, \omega, \mu)$ defines a graph $\Gamma:=\Gamma(M, \omega, \mu)$, called a GKM graph, where the vertex set and the oriented edge set are defined as follows:

- the vertex set $V_{\Gamma}$ is equal to $M^{T}$,

- the oriented edge set $E_{\Gamma}$ consists of pairs $(p, q) \in V_{\Gamma} \times V_{\Gamma}(p \neq q)$ such that $p$ and $q$ are in the same component of the $H$-fixed point set $M^{H}$ for some codimension one subtorus $H$ of $T$. Equivalently, $(p, q) \in E_{\Gamma}$ if and only if $p$ and $q$ are contained in a $T$-invariant two-sphere in $M$. In particular, $(p, q) \in E_{\Gamma}$ if and only if $(q, p) \in E_{\Gamma}$.

We call the two conditions (1) and (2) in Definition 3.1 the GKM conditions. Note that each $v \in V_{\Gamma}$ is contained in exactly $n$ edges, i.e., $\Gamma$ is an $n$-valent graph. Indeed, for each fixed point $v \in M^{T}$, the tangential $T$-representation on $T_{v} M$ splits into the sum of one-dimensional irreducible representations so that

$$
T_{v} M=\oplus_{j=1}^{n} \xi_{j}
$$


where $\xi_{j}$ is a one-dimensional irreducible $T$-representation with weight $\alpha_{j, v} \in \mathfrak{t}^{*}$ for $j=1, \cdots, n$. Then any element $z_{j} \in \xi_{j} \subset T_{v} M$ is fixed by the adjoint action of ker $\alpha_{j, v}$, and therefore $\xi_{j}$ is fixed by the codimension one subtorus $H_{j}:=\exp \left(\operatorname{ker} \alpha_{j, v}\right)$ of $T$. By the second GKM condition (2), the connected component of $M^{H_{j}}$ containing $v$ is of dimension two, i.e., it is a two-sphere and it contains exactly two fixed points, say $v$ and $v_{j}^{\prime}$, of the $T$-action. Thus there exist $n$ fixed points $v_{1}^{\prime}, \cdots, v_{n}^{\prime}$ of the $T$-action such that $\left(v, v_{j}^{\prime}\right) \in E_{\Gamma}$ for each $j=1, \cdots, n$. Furthermore, the GKM condition (2) implies that there is no more edge containing $v$ except for $\left(v, v_{j}^{\prime}\right)$ 's for $j=1, \cdots, n$.

For an oriented edge $e=(p, q) \in E_{\Gamma}$, we denote by $i(e)$ and $t(e)$ the initial vertex $p$ and the terminal vertex $q$ of $e$, respectively. For each $\xi \in \mathfrak{t}$, let $\mu_{\xi}:=\langle\mu, \xi\rangle$ where $\langle$,$\rangle is the canonical pairing of \mathfrak{t}^{*}$ and $\mathfrak{t}$. We say $\xi$ is generic if

$$
\mu_{\xi}(i(e)) \neq \mu_{\xi}(t(e))
$$

for every $e \in E_{\Gamma}$. In other words, $\xi$ is generic if $\xi$ is not perpendicular to $\mu(q)-\mu(p)$ for any edge $(p, q)$ of $\Gamma$.

Now, fix a generic $\xi \in \mathfrak{t}$. We say that $e \in E_{\Gamma}$ is ascending (resp. descending) with respect to $\xi$ if $\mu_{\xi}(i(e))<\mu_{\xi}(t(e))$ (resp. $\left.\mu_{\xi}(i(e))>\mu_{\xi}(t(e))\right)$. The index of $v \in V_{\Gamma}$, denoted by $\lambda_{v}$, is defined as twice the number of descending edges starting at $v$.

Remark 3.2. We can always take a generic element $\xi$ lying on the lattice of $\mathfrak{t}$ so that $\xi$ generates a circle subgroup $S^{1}$ of $T$. Then the Hamiltonian $S^{1}$-action generated by $\xi$ has a moment map $\mu_{\xi}=\langle\xi, \mu\rangle$ and the genericity of $\xi$ implies that the fixed point set $M^{S^{1}}$ for the $S^{1}$-action is the same as $M^{T}$. Moreover, $\mu_{\xi}$ is a Morse function on $M$ such that each fixed point $v \in M^{S^{1}}$ has a Morse index equal to $\lambda_{v}$. See [Au] for more details.

Definition 3.3. Let $\xi \in \mathfrak{t}$ be a generic vector. $\Gamma$ is called index increasing with respect to $\xi \in \mathfrak{t}$ if

$$
\mu_{\xi}(i(e))<\mu_{\xi}(t(e)) \quad \text { implies } \quad \lambda_{i(e)}<\lambda_{t(e)}
$$

for every $e \in E_{\Gamma}$. If $\Gamma$ is index increasing with respect to some $\xi \in \mathfrak{t}$, then $\Gamma$ is simply called index increasing.

Remark 3.4. We note that if $\Gamma$ is index increasing with respect to $\xi \in \mathfrak{t}$, then $\Gamma$ is also index increasing with respect to $-\xi$.

3.2. Graph cohomology rings. For each $e \in E_{\Gamma}$, we denote by $S_{e}^{2}$ the unique $T$-invariant two-sphere containing $i(e)$ and $t(e)$. Let us define a function $\alpha$, called an axial function of $\Gamma$, which assigns the weight of the one-dimensional tangential $T$-representation on $T_{i(e)} S_{e}^{2}$ for each $e \in E_{\Gamma}$ :

$$
\alpha: E_{\Gamma} \longrightarrow \mathfrak{t}^{*}, \quad e \longmapsto \alpha(e) .
$$

Notation 3.5. For the sake of simplicity, we denote by $(p, q)$ the oriented edge $e$ such that $i(e)=p$ and $t(e)=q$. Also, we denote $\alpha((p, q))$ by $\alpha(p, q)$.

Definition 3.6. For a given pair $(\Gamma, \alpha)$, the graph cohomology ring $H(\Gamma, \alpha)$ is defined by

$$
\left\{h: V_{\Gamma} \rightarrow \mathrm{S}\left(\mathfrak{t}^{*}\right) \mid h(t(e))-h(i(e)) \equiv 0 \quad \bmod \alpha(e) \text { for every } e \in E_{\Gamma}\right\},
$$

where $\mathrm{S}\left(\mathfrak{t}^{*}\right)$ is identified with a polynomial ring $\mathbb{R}\left[x_{1}, \cdots, x_{m}\right]$ as in $(2.1)$.

The product structure on $H(\Gamma, \alpha)$ is defined by

$$
\left(h_{1} \cdot h_{2}\right)(v):=h_{1}(v) h_{2}(v) \in S\left(\mathfrak{t}^{*}\right) \cong \mathbb{R}\left[x_{1}, \cdots, x_{m}\right]
$$

for every $h_{1}, h_{2} \in H(\Gamma, \alpha)$. The graph cohomology ring $H(\Gamma, \alpha)$ has a natural $\mathbb{Z}$-grading given by

$$
H^{i}(\Gamma, \alpha):=H(\Gamma, \alpha) \cap \operatorname{Map}\left(V_{\Gamma}, \mathrm{S}^{i}\left(\mathfrak{t}^{*}\right)\right)
$$

where $\mathrm{S}^{i}\left(\mathfrak{t}^{*}\right)$ is the $\mathbb{R}$-subspace of $\mathrm{S}\left(\mathfrak{t}^{*}\right)$ generated by $i$-times symmetric tensor products of elements in $\mathfrak{t}^{*}$ for $i>0$. When $i=0$, we put $\mathrm{S}^{0}\left(\mathfrak{t}^{*}\right)=\mathbb{R}$. 
Together with the product structure, $H(\Gamma, \alpha)$ becomes a commutative $\mathbb{Z}$-graded ring. Also, any $\mathrm{S}\left(\mathfrak{t}^{*}\right)$ valued constant function on $V_{\Gamma}$ is an element of $H(\Gamma, \alpha)$ and hence $\mathrm{S}\left(\mathfrak{t}^{*}\right)$ is a subring of $H(\Gamma, \alpha)$. Therefore, $H(\Gamma, \alpha)$ is an $\mathrm{S}\left(\mathfrak{t}^{*}\right)$-algebra with the unit $1 \in \mathrm{S}^{0}\left(\mathfrak{t}^{*}\right)=\mathbb{R}$.

Lemma 3.7. Let $\Gamma$ and $\alpha$ be given as above.

(1) Let $h \in H^{1}(\Gamma, \alpha)$ and $e \in E_{\Gamma}$. If $h(t(e))=0$, then $h(i(e))=k \cdot \alpha(e)$ for some $k \in \mathbb{R}$.

(2) Let $h \in H^{i}(\Gamma, \alpha)$ for some $i \leq n-1$. If $h(v)=0$ for every vertex $v$ except one, then $h=0$.

Proof. (1) is straightforward by definition of $H(\Gamma, \alpha)$. For $(2)$, assume that $h\left(v_{0}\right) \neq 0$ for some $v_{0} \in V_{\Gamma}$ and $h(v)=0$ for any other vertex $v \neq v_{0}$. Then $\alpha\left(v, v_{0}\right)$ divides $h\left(v_{0}\right)$ for every $v$ adjacent to $v_{0}$. Also, these $\alpha\left(v, v_{0}\right)$ 's are pairwise linearly independent by the GKM condition (2). Thus $h\left(v_{0}\right)$ should be of polynomial degree at least $n$ since $\mathrm{S}\left(\mathfrak{t}^{*}\right)$ is a UFD. This contradicts that $\operatorname{deg} h\left(v_{0}\right) \leq n-1$, and therefore $h\left(v_{0}\right)=0$.

3.3. Equivariant Thom classes. Let $\xi \in \mathfrak{t}$ be a generic vector. A path of $\Gamma$ is a sequence of vertices $\left(v_{0}, \cdots, v_{l}\right)$ of $\Gamma$ such that $\left(v_{j}, v_{j+1}\right) \in E_{\Gamma}$ for every $j=0, \cdots, l-1$. We say that a path $\left(v_{0}, \cdots, v_{l}\right)$ is ascending (resp. descending) with respect to $\xi$ if each $\left(v_{j}, v_{j+1}\right)$ is ascending (resp. descending) with respect to $\xi$ for every $j$.

For each $v \in V_{\Gamma}$, let $E_{v}^{\uparrow}$ (resp. $E_{v}^{\downarrow}$ ) be the set of ascending (resp. descending) edges with respect to $\xi$ having the initial vertex $v$. Note that $\left|E_{v}^{\downarrow}\right|=\lambda_{v} / 2$ where $\lambda_{v}$ is the index of $v$ (with respect to $\xi$ ) and $\lambda_{v}$ is equal to the Morse index of $v$ with respect to $\mu_{\xi}$, see Remark 3.2.

For each $h \in H(\Gamma, \alpha)$, define a support of $h$ by

$$
\operatorname{supp} h:=\left\{v \in V_{\Gamma} \mid h(v) \neq 0\right\}
$$

Guillemin-Zara [GZ] proved that there exists a nice basis of $H(\Gamma, \alpha)$ as an $\mathrm{S}\left(\mathfrak{t}^{*}\right)$-module whose elements are called equivariant Thom classes.

Theorem 3.8. [GZ, Theorem 1.5, 1.6] Let $(M, \omega, \mu)$ be a Hamiltonian GKM manifold with its GKM graph $\left(\Gamma, V_{\Gamma}, E_{\Gamma}\right)$. If $\Gamma$ is index increasing with respect to some generic $\xi \in \mathfrak{t}$, then for each $v \in V_{\Gamma}$, there exists a unique element $\tau_{v}^{+}$of $H^{\lambda_{v} / 2}(\Gamma, \alpha)$ satisfying

(1) $\operatorname{supp} \tau_{v}^{+} \subset\left\{v^{\prime} \in V_{\Gamma} \mid\right.$ there exists an ascending path with respect to $\xi$ from $v$ to $\left.v^{\prime}\right\}$, and

(2) $\tau_{v}^{+}(v)=\Lambda_{v}^{+}:=\prod_{e \in E_{v}^{\downarrow}} \alpha(e)$.

Furthermore, the set $\left\{\tau_{v}^{+}\right\}_{v \in V_{\Gamma}}$ forms a basis of $H(\Gamma, \alpha)$ as an $\mathrm{S}\left(\mathfrak{t}^{*}\right)$-module.

We call $\tau_{v}^{+}$the equivariant Thom class for $v \in V_{\Gamma}$ with respect to $\xi$. As in Remark 3.4, if $\Gamma$ is index increasing with respect to $\xi$, then $\Gamma$ is also index increasing with respect to $-\xi$. We denote by $\tau_{v}^{-}$the equivariant Thom class for $v \in V_{\Gamma}$ with respect to $-\xi$. Then by Theorem 3.8, we have

$$
\tau_{v}^{-}(v)=\Lambda_{v}^{-}:=\prod_{e \in E_{v}^{\uparrow}} \alpha(e) \quad \text { and } \quad \Lambda_{v}=\Lambda_{v}^{+} \cdot \Lambda_{v}^{-},
$$

where $\Lambda_{v}$ is the equivariant Euler class of the normal bundle to $v$ in $M$.

3.4. GKM description of equivariant cohomology. Recall that the inclusion map $\imath: M^{T} \hookrightarrow M^{T}$ induces an $H^{*}(B T)$-algebra homomorphism

$$
\imath^{*}: H_{T}^{*}(M) \rightarrow H_{T}^{*}\left(M^{T}\right) \cong \bigoplus_{v \in M^{T}} H_{T}^{*}(\{v\}) .
$$

In particular, for each fixed point $v \in M^{T}$, the inclusion $\imath_{v}:\{v\} \hookrightarrow M$ induces the map

$$
\imath_{v}^{*}: H_{T}^{*}(M) \rightarrow H_{T}^{*}(\{v\}) \cong H^{*}(\{v\}) \otimes H^{*}(B T) \cong H^{*}(B T),
$$

and the image $\imath_{v}^{*}(\beta)$ is called the restriction of $\beta$ to $v$ and is denoted by $\left.\beta\right|_{v}$ for every $\beta \in H_{T}^{*}(M)$. See Section 2.2. The following theorem is a symplectic version of the theorem [GKM] due to Goresky, Kottwitz, and MacPherson, which enables us to identify $H_{T}^{*}(M)$ with $H(\Gamma, \alpha)$. 
Theorem 3.9. [GKM] Let $(M, \omega, \mu)$ be a closed Hamiltonian GKM manifold with its GKM graph $\left(\Gamma, V_{\Gamma}, E_{\Gamma}\right)$. Then the map

$$
H_{T}^{*}(M) \longrightarrow H(\Gamma, \alpha), \quad \beta \longmapsto h_{\beta}
$$

is an $\mathrm{S}\left(\mathfrak{t}^{*}\right)$-algebra isomorphism where

$$
h_{\beta}(v):=\left.\beta\right|_{v}
$$

for each $v \in V_{\Gamma}$. The image of $H_{T}^{2 l}(M)$ under this isomorphism is $H^{l}(\Gamma, \alpha)$ for every integer $l \geq 0$.

Let $e \in E_{\Gamma}$ be any oriented edge. Let us label the $n$ edges outward from $i(e)$ by $e_{1, i(e)}, \cdots, e_{n, i(e)}$. Also, let

$$
\alpha_{j, i(e)}:=\alpha\left(i\left(e_{j, i(e)}\right), t\left(e_{j, i(e)}\right)\right)=\alpha\left(i(e), t\left(e_{j, i(e)}\right)\right) .
$$

Also, we can define $\alpha_{j, t(e)}$ 's in a similar way.

Lemma 3.10. [GZ, Proposition 2.2] For each oriented edge $e \in E_{\Gamma}$, we can reorder $e_{j, i(e)}$ 's and $e_{j, t(e)}$ 's so that

$$
\alpha_{n, t(e)}=-\alpha_{n, i(e)}=-\alpha(e) \quad \text { and } \quad \alpha_{j, t(e)} \equiv \alpha_{j, i(e)} \bmod \alpha(e)
$$

for each $1 \leq j \leq n-1$.

\section{Hodge-Riemann BILINEAR Forms}

Let $(M, \omega)$ be a $2 n$-dimensional closed symplectic manifold and let $T$ be an $m$-dimensional $(m \geq 2)$ compact torus acting on $(M, \omega)$ in a Hamiltonian fashion with a moment map $\mu: M \rightarrow \mathfrak{t}^{*}$. Assume the action is GKM and the corresponding GKM graph $\Gamma$ is index increasing with respect to some generic vector $\xi \in \mathfrak{t}^{*}$ so that the equivariant Thom class exists for every vertex $v \in V_{\Gamma}$ by Theorem 3.8. In the section, we compute the matrix $A_{l}(M, \omega)$ presenting the Hodge-Riemann bilinear form $\mathrm{HR}_{l}$ for each $l=0, \cdots, n$.

For a fixed $l$ with $0 \leq l \leq n$, let $b_{l}:=b_{l}(M)$ be the $l$-th Betti number of $M$ and let

$$
\left\{p_{1}, \cdots, p_{b_{l}}\right\} \quad \text { and } \quad\left\{q_{1}, \cdots, q_{b_{l}}\right\}
$$

be the set of vertices of index $l$ and index $2 n-l$, respectively. Then Theorem 3.8 and Theorem 2.3 imply that each of

$$
\mathcal{B}_{l}^{+}:=\left\{f^{*} \tau_{p_{1}}^{+}, \cdots, f^{*} \tau_{p_{b_{l}}}\right\} \quad \text { and } \quad \mathcal{B}_{l}^{-}:=\left\{f^{*} \tau_{q_{1}}^{-}, \cdots, f^{*} \tau_{q_{b_{l}}}^{-}\right\}
$$

forms a basis of $H^{l}(M)$ where $f: M \hookrightarrow M \times_{T} E T$ is an inclusion of a fiber $M$, see (2.2). Then the Hodge-Riemann form $\mathrm{HR}_{l}$ is represented by the following $b_{l} \times b_{l}$ matrix

$$
A_{l}(M, \omega)=\left(a_{j k}\right)_{1 \leq j, k \leq b_{l}}:=\left(\operatorname{HR}_{l}\left(f^{*} \tau_{p_{k}}^{+}, f^{*} \tau_{q_{j}}^{-}\right)\right)_{1 \leq j, k \leq b_{l}} .
$$

It is straightforward that $(M, \omega)$ satisfies the hard Lefschetz property if and only if $A_{l}(M, \omega)$ is non-singular for every $l=0,1, \cdots, n$.

4.1. $\Theta$ function and vol function. To compute each entry $a_{j k}$ of $A_{l}(M, \omega)$, we define two functions $\Theta$ and vol as follows. The function vol, called the volume function, is defined by

$$
\operatorname{vol}: E_{\Gamma} \longrightarrow \mathbb{R}, \quad(p, q) \longmapsto(\mu(q)-\mu(p)) / \alpha(p, q)
$$

for every $(p, q) \in E_{\Gamma}$.

Lemma 4.1. For any $(p, q) \in E_{\Gamma}$, the symplectic area of the T-invariant two-sphere $S_{(p, q)}^{2}$ containing $p$ and $q$ is equal to $\operatorname{vol}(p, q)$. In particular, $\operatorname{vol}(p, q)$ is a positive real number. 
Proof. Let $i: S_{(p, q)}^{2} \hookrightarrow M$ be the embedding of $S_{(p, q)}^{2}$ into $M$. Then the symplectic volume $\int_{S_{(p, q)}^{2}} i^{*} \omega$ is equal to the integration along the fiber $\int_{S_{(p, q)}^{2}} i^{*}\left[\widetilde{\omega}_{\mu}\right]$ where $\left[\widetilde{\omega}_{\mu}\right] \in H_{T}^{2}(M)$ is the equivariant symplectic class with respect to $\mu$. By the ABBV-localization theorem (Theorem 2.6) and Lemma 2.7, we have

$$
\int_{S_{(p, q)}^{2}} i^{*}\left[\widetilde{\omega}_{\mu}\right]=\frac{\left.\left[\widetilde{\omega}_{\mu}\right]\right|_{p}}{e_{p}}+\frac{\left.\left[\widetilde{\omega}_{\mu}\right]\right|_{q}}{e_{q}}=\frac{-\mu(p)}{\alpha(p, q)}+\frac{-\mu(q)}{\alpha(q, p)}=\frac{\mu(q)-\mu(p)}{\alpha(p, q)} .
$$

This completes the proof.

Now, following [GT, p. 453], we define the function $\Theta$ by

$$
\begin{aligned}
\Theta: E_{\Gamma} & \rightarrow Q\left(\mathfrak{t}^{*}\right) \\
(p, q) & \mapsto \Theta(p, q):=\frac{\rho_{\alpha(p, q)}\left(\Lambda_{p}^{+}\right)}{\rho_{\alpha(p, q)}\left(\Lambda_{q}^{+} / \alpha(q, p)\right)}
\end{aligned}
$$

where $Q\left(\mathfrak{t}^{*}\right)$ is the quotient field of $\mathrm{S}\left(\mathfrak{t}^{*}\right)$ and $\rho_{\alpha(p, q)}$ is the canonical extension of the projection map

$$
X \mapsto X-\frac{\langle X, \xi\rangle}{\langle\alpha(p, q), \xi\rangle} \alpha(p, q) \quad \text { on } \mathfrak{t}^{*}
$$

to $\mathrm{S}\left(\mathfrak{t}^{*}\right)$. Note that $\Theta(p, q) \in Q\left(\mathfrak{t}^{*}\right)$ is a nonzero element (rational function) in $Q\left(\mathfrak{t}^{*}\right)$ by the GKM conditions. Moreover, it has an integer value when $\lambda_{q}-\lambda_{p}=2$. See [ST, Theorem 2.4].
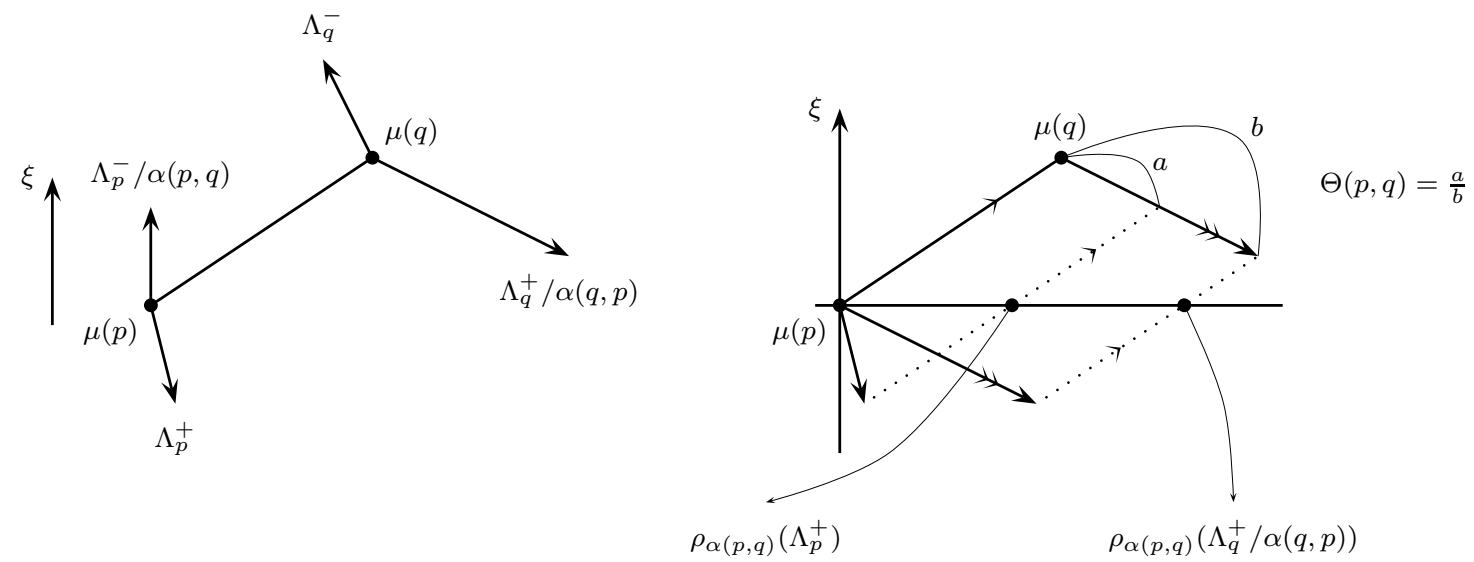

Figure 4.1. Goldin-Tolman's $\Theta$ function

Lemma 4.2. $\rho_{\alpha(p, q)}=\rho_{\alpha(q, p)}$ for every $(p, q) \in E_{\Gamma}$.

Proof. It is straightforward by definition of $\rho$.

On the other hand, let us consider $\Gamma$ with an opposite generic vector $-\xi \in \mathfrak{t}^{*}$. Then $\Gamma$ is also indexincreasing with respect to $-\xi$ and the index of $v$, denoted by $\bar{\lambda}_{v}$, with respect to $\mu_{-\xi}$ is given by $\bar{\lambda}_{v}=2 n-\lambda_{v}$ for every $v \in V_{\Gamma}$. Let $\bar{\Theta}$ be the Goldin-Tolman's $\Theta$ function with respect to $-\xi$ so that

$$
\bar{\Theta}(q, p)=\frac{\rho_{\alpha(q, p)}\left(\Lambda_{q}^{-}\right)}{\rho_{\alpha(q, p)}\left(\Lambda_{p}^{-} / \alpha(p, q)\right)} .
$$

By applying Lemma 4.2, we have

$$
\begin{aligned}
\frac{\Theta(p, q)}{\bar{\Theta}(q, p)} & =\frac{\rho_{\alpha(q, p)}\left(\Lambda_{p}^{-} / \alpha(p, q)\right)}{\rho_{\alpha(q, p)}\left(\Lambda_{q}^{-}\right)} \cdot \frac{\rho_{\alpha(p, q)}\left(\Lambda_{p}^{+}\right)}{\rho_{\alpha(p, q)}\left(\Lambda_{q}^{+} / \alpha(q, p)\right)} \\
& =\frac{\rho_{\alpha(p, q)}\left(\Lambda_{p} / \alpha(p, q)\right)}{\rho_{\alpha(p, q)}\left(\Lambda_{q} / \alpha(q, p)\right)} .
\end{aligned}
$$


Lemma 4.3. For any $(p, q) \in E_{\Gamma}$, we have

$$
\frac{\rho_{\alpha(p, q)}\left(\Lambda_{p} / \alpha(p, q)\right)}{\rho_{\alpha(p, q)}\left(\Lambda_{q} / \alpha(q, p)\right)}=1
$$

and therefore $\Theta(p, q)=\bar{\Theta}(q, p)$.

Proof. Without loss of generality, we may assume that $(p, q)$ is ascending with respect to $\xi$. By Lemma 3.10 , we can give orders on the set of edges $\left\{e_{1}, \cdots, e_{n}\right\}$ having initial vertex $p$ and on $\left\{e_{1}^{\prime}, \cdots, e_{n}^{\prime}\right\}$ having initial vertex $q$ such that

- $\alpha\left(e_{n}\right)=-\alpha\left(e_{n}^{\prime}\right)=\alpha(p, q)$, and

- $\alpha\left(e_{j}\right)=\alpha\left(e_{j}^{\prime}\right)+c_{j} \alpha\left(e_{n}\right)=\alpha\left(e_{j}^{\prime}\right)+c_{j} \alpha(p, q)$ for some $c_{j} \in \mathbb{R}$

for every $j=1, \cdots, n-1$. Then $\alpha\left(e_{1}\right) \cdots \alpha\left(e_{n-1}\right)=\alpha\left(e_{1}^{\prime}\right) \cdots \alpha\left(e_{n-1}^{\prime}\right)$ modulo $\alpha(p, q)$ in $\mathrm{S}\left(\mathfrak{t}^{*}\right)$. Since $\alpha(p, q)$ is in the kernel of $\rho_{\alpha(p, q)}$, we have $\rho_{\alpha(p, q)}\left(\alpha\left(e_{1}\right) \cdots \alpha\left(e_{n-1}\right)\right)=\rho_{\alpha(p, q)}\left(\alpha\left(e_{1}^{\prime}\right) \cdots \alpha\left(e_{n-1}^{\prime}\right)\right)$. Furthermore, the GKM conditions imply that $\rho_{\alpha(p, q)}\left(\alpha\left(e_{j}\right)\right) \neq 0$ and $\rho_{\alpha(p, q)}\left(\alpha\left(e_{j}^{\prime}\right)\right) \neq 0$ for every $j=1, \cdots, n-1$. Therefore,

$$
\begin{aligned}
\rho_{\alpha(p, q)}\left(\Lambda_{p} / \alpha(p, q)\right) & =\rho_{\alpha(p, q)}\left(\alpha\left(e_{1}\right) \cdots \alpha\left(e_{n-1}\right)\right)=\rho_{\alpha(p, q)}\left(\alpha\left(e_{1}^{\prime}\right) \cdots \alpha\left(e_{n-1}^{\prime}\right)\right) \\
& =\rho_{\alpha(p, q)}\left(\Lambda_{q} / \alpha(q, p)\right) \neq 0 .
\end{aligned}
$$

This completes the proof.

4.2. Computation of $A_{l}(M, \omega)$. Let $\mathbf{v}=\left(v_{0}, v_{1}, \cdots, v_{s}\right)$ be an ascending path of $\Gamma$ with respect to a generic $\xi$. Following [GT], the length of $\mathbf{v}$ is defined to be $s$ and denoted by $|\mathbf{v}|$. For any two vertices $p$ and $q$ in $V_{\Gamma}$, let $\sum_{p}^{q}$ be the set of ascending paths from $p$ to $q$ :

$$
\left\{\mathbf{v}=\left(v_{0}, v_{1}, \cdots, v_{|\mathbf{v}|}\right) \mid v_{0}=p, v_{|\mathbf{v}|}=q, \lambda_{v_{j+1}}-\lambda_{v_{j}}=2 \text {, and }\left(v_{j}, v_{j+1}\right) \in E_{\Gamma} \text { for any } 0 \leq j \leq|\mathbf{v}|-1\right\}
$$

where $|\mathbf{v}|=\left(\lambda_{q}-\lambda_{p}\right) / 2$. Also, we denote by $\sum_{p}^{q}(r)$ the subset of $\sum_{p}^{q}$ consisting of paths passing through $r$ for $r \in V_{\Gamma}$.

Now, for $1 \leq l \leq n$, let $A_{l}(M, \omega)$ be the $\left(b_{l} \times b_{l}\right)$-matrix with respect to the bases $\mathcal{B}_{l}^{+}$and $\mathcal{B}_{l}^{-}$defined in (4.1). The following proposition states that each entry of $A_{l}(M, \omega)$ can be computed by using vol, $\Theta$, and $\mu$.

Proposition 4.4. The $(j, k)$-th entry $a_{j k}$ of $A_{l}(M, \omega)$ is equal to

$$
\sum_{r \in V_{\Gamma}}\left[\prod_{i=1}^{n-l}\left[\mu(r)-d_{i}\right]\right] \cdot\left[\sum_{\mathbf{v} \in \sum_{p_{k}}^{q_{j}}(r)} \frac{\prod_{i=1}^{n-l}\left[\operatorname{vol}\left(v_{i-1}, v_{i}\right) \cdot \Theta\left(v_{i-1}, v_{i}\right)\right]}{\prod_{i \in\{0,1, \cdots, n-l\} \backslash\left\{c_{r}\right\}}\left[\mu(r)-\mu\left(v_{i}\right)\right]}\right]
$$

for $1 \leq j, k \leq b_{l}$ where $d_{1}, \cdots, d_{n-l}$ are any elements in $\mathbf{t}^{*}$ and $c_{r}=\left(\lambda_{r}-\lambda_{p_{k}}\right) / 2$.

To prove Proposition 4.4, we use the following theorem due to [GT]. (More general formula can be found in $[\mathrm{ST}]$.)

Theorem 4.5. [GT, Theorem 1.6] For any $p, q \in V_{\Gamma}$, the following holds:

$$
\tau_{p}^{+}(q)=\Lambda_{q}^{+} \cdot \sum_{\mathbf{v} \in \sum_{p}^{q}} \prod_{i=1}^{|\mathbf{v}|} \frac{\mu\left(v_{i}\right)-\mu\left(v_{i-1}\right)}{\mu(q)-\mu\left(v_{i-1}\right)} \cdot \frac{\Theta\left(v_{i-1}, v_{i}\right)}{\alpha\left(v_{i}, v_{i-1}\right)},
$$

where $\mathbf{v}=\left(p=v_{0}, v_{1}, \cdots, v_{|\mathbf{v}|}=q\right)$.

Remark 4.6. In $[\mathrm{GT}]$, they used the opposite sign convention to ours. For example, our $\alpha(p, q)$ should be $\alpha(q, p)$ in [GT] and our $\Lambda_{p}^{+}$should be $\Lambda_{p}^{-}$in [GT]. Note that the notation $\eta(p, q)$ used in [GT] is the same as $\alpha(q, p)$. Also, $\alpha_{p}$ (the canonical class) in [GT] is the same as $\tau_{p}^{+}$in our paper. 
Proof of Proposition 4.4. Let us fix $k$ and $j$ with $1 \leq k, j \leq b_{l}$. By Theorem 4.5, we have

$$
\tau_{p_{k}}^{+}(r)=\Lambda_{r}^{+} \cdot \sum_{\mathbf{v} \in \sum_{p_{k}}^{r}} \prod_{i=1}^{|\mathbf{v}|} \frac{\mu\left(v_{i}\right)-\mu\left(v_{i-1}\right)}{\mu(r)-\mu\left(v_{i-1}\right)} \cdot \frac{\Theta\left(v_{i-1}, v_{i}\right)}{\alpha\left(v_{i}, v_{i-1}\right)}
$$

for every vertex $r \in V_{\Gamma}$. Note that the length of $\mathbf{v} \in \Sigma_{p_{k}}^{r}$ is $\frac{\lambda_{r}-\lambda_{p_{k}}}{2}$, which we denote by $c_{r}$. By substituting

$$
\operatorname{vol}\left(v_{i-1}, v_{i}\right)=\frac{\mu\left(v_{i}\right)-\mu\left(v_{i-1}\right)}{\alpha\left(v_{i-1}, v_{i}\right)}
$$

to the the above formula, we have

$$
\tau_{p_{k}}^{+}(r)=\Lambda_{r}^{+} \cdot \sum_{\mathbf{v} \in \sum_{p_{k}}^{r}} \prod_{i=1}^{|\mathbf{v}|} \frac{\operatorname{vol}\left(v_{i-1}, v_{i}\right) \cdot \Theta\left(v_{i-1}, v_{i}\right)}{-\mu(r)+\mu\left(v_{i-1}\right)} .
$$

Similarly, with respect to $-\xi \in \mathfrak{t}$, we have

$$
\tau_{q_{j}}^{-}(r)=\Lambda_{r}^{-} \cdot \sum_{\mathbf{v} \in \sum_{r}^{q_{j}}} \prod_{i=1}^{|\mathbf{v}|} \frac{\operatorname{vol}\left(v_{i-1}, v_{i}\right) \cdot \bar{\Theta}\left(v_{i}, v_{i-1}\right)}{-\mu(r)+\mu\left(v_{i}\right)}
$$

for every $r \in V_{\Gamma}$ by Lemma 4.3. Therefore, we have

$$
\tau_{p_{k}}^{+}(r) \cdot \tau_{q_{j}}^{-}(r)=\Lambda_{r} \cdot \sum_{\mathbf{v} \in \sum_{p_{k}}^{q_{j}}(r)} \frac{\prod_{i=1}^{n-l} \operatorname{vol}\left(v_{i-1}, v_{i}\right) \cdot \Theta\left(v_{i-1}, v_{i}\right)}{\prod_{i \in\{0,1, \cdots, n-l\} \backslash\left\{c_{r}\right\}}\left[-\mu(r)+\mu\left(v_{i}\right)\right]},
$$

since $|\mathbf{v}|=n-l$ for every $\mathbf{v} \in \Sigma_{p_{k}}^{q_{j}}(r)$ and each $\mathbf{v}$ is of the form

$$
\mathbf{v}=\left(v_{0}=p_{k}, \cdots, v_{c_{r}}=r, \cdots, v_{n-l}=q_{j}\right) .
$$

Eventually, by applying the ABBV-localization theorem (Theorem 2.6), we have

$$
\begin{aligned}
a_{j k} & =\left\langle[\omega]^{n-l} \wedge f^{*}\left(\tau_{p_{k}}^{+}\right) \wedge f^{*}\left(\tau_{q_{j}}^{-}\right),[M]\right\rangle \\
& =\int_{M}\left[\prod_{i=1}^{n-l}\left[\widetilde{\omega}_{i}\right]\right] \cdot \tau_{p_{k}}^{+} \cdot \tau_{q_{j}}^{-} \\
& =\sum_{r \in V_{\Gamma}}\left(\left[\prod_{i=1}^{n-l}\left[\widetilde{\omega}_{i}\right]\right] \cdot \tau_{p_{k}}^{+} \cdot \tau_{q_{j}}^{-}\right)(r) / \Lambda_{r},
\end{aligned}
$$

where $\widetilde{\omega}_{i}$ is any equivariant symplectic form for each $i=1, \cdots, n-l$. Note that (4.5) is equal to

$$
\sum_{r \in V_{\Gamma}}\left[\prod_{i=1}^{n-l}\left[\widetilde{\omega}_{i}\right]\right](r) \cdot\left[\sum_{\mathbf{v} \in \sum_{p_{k}}^{q_{j}}(r)} \frac{\prod_{i=1}^{n-l} \operatorname{vol}\left(v_{i-1}, v_{i}\right) \cdot \Theta\left(v_{i-1}, v_{i}\right)}{\prod_{i \in\{0,1, \cdots, n-l\} \backslash\left\{c_{r}\right\}}\left[-\mu(r)+\mu\left(v_{i}\right)\right]}\right]
$$

by (4.4). Also, note that $\left[\widetilde{\omega}_{i}\right](r)=\left.\left[\widetilde{\omega}_{i}\right]\right|_{r}=-\mu(r)+d_{i}$ for some $d_{i} \in \mathfrak{t}^{*}$. Since (4.5) holds for any choice of $\omega_{i}$, each $d_{i}$ can be chosen arbitrarily. Therefore, the coefficient $a_{j k}$ is equal to

$$
\sum_{r \in V_{\Gamma}}\left[\prod_{i=1}^{n-l}\left[\mu(r)-d_{i}\right]\right] \cdot\left[\sum_{\mathbf{v} \in \sum_{P_{k}}^{q_{j}}(r)} \frac{\prod_{i=1}^{n-l}\left[\operatorname{vol}\left(v_{i-1}, v_{i}\right) \cdot \Theta\left(v_{i-1}, v_{i}\right)\right]}{\prod_{i \in\{0,1, \cdots, n-l\} \backslash\left\{c_{r}\right\}}\left[\mu(r)-\mu\left(v_{i}\right)\right]}\right] .
$$

This finishes the proof.

From Proposition 4.4, we can obtain the following.

Corollary 4.7. Suppose that $n-l=1$. Then

(1) $a_{j k}= \begin{cases}\Theta\left(p_{k}, q_{j}\right) \cdot \operatorname{vol}\left(p_{k}, q_{j}\right) & \text { if }\left(p_{k}, q_{j}\right) \in E_{\Gamma}, \\ 0 & \text { if }\left(p_{k}, q_{j}\right) \notin E_{\Gamma}, \text { and }\end{cases}$

(2) $a_{j k}$ is nonzero if and only if $\left(p_{k}, q_{j}\right) \in E_{\Gamma}$. 
Proof. Suppose that $n-l=1$ and let $p=p_{k}$ (resp. $q=q_{j}$ ) be any index $l$ (resp. index $2 n-l$ ) vertex in $V_{\Gamma}$. If $p$ and $q$ are not adjacent, then $a_{j k}=0$ by Proposition 4.4 since $\sum_{p}^{q}$ is empty. If $p$ and $q$ are adjacent, the formula of Proposition 4.4 is reduced to

$$
\begin{aligned}
a_{j k} & =(\mu(p)-d) \cdot \frac{\operatorname{vol}(p, q) \cdot \Theta(p, q)}{\mu(p)-\mu(q)}+(\mu(q)-d) \cdot \frac{\operatorname{vol}(p, q) \cdot \Theta(p, q)}{\mu(q)-\mu(p)} \\
& =\operatorname{vol}(p, q) \cdot \Theta(p, q)
\end{aligned}
$$

for any choice of $d \in \mathfrak{t}^{*}$. The second statement (2) easily follows from (1) and the fact that $\operatorname{vol}(p, q)$ and $\Theta(p, q)$ are both nonzero for every $(p, q) \in E_{\Gamma}$.

4.3. Concluding remark. Sabatini and Tolman [ST, Theorem 0.3] gave a generalized formula of Theorem 4.5. We state the modified version of the theorem which fits in our context as follows.

Theorem 4.8. [ST] Let $(M, \omega, \mu)$ be a Hamiltonian GKM T-manifold such that the corresponding GKM graph $\Gamma$ is index increasing with respect to some generic vector $\xi \in \mathfrak{t}$. Let $p$ and $q$ be any two fixed point. For each fixed point $z \in M^{T}$, let $w_{z}$ be any element in $H_{T}^{2}(M)$ such that $w_{z}(q) \neq w_{z}(z)$. Then

$$
\tau_{p}^{+}(q)=\Lambda_{q}^{+} \cdot\left[\sum_{\mathbf{v} \in \sum_{p}^{q}} \prod_{i=1}^{|\mathbf{v}|} \frac{w_{v_{i}}\left(v_{i+1}\right)-w_{v_{i}}\left(v_{i}\right)}{w_{v_{i}}(q)-w_{v_{i}}\left(v_{i}\right)} \cdot \frac{\tau_{v_{i}}^{+}\left(v_{i+1}\right)}{\Lambda_{v_{i+1}}^{+}}\right] .
$$

Remark 4.9. We can easily see that Theorem 4.8 is a generalization of Theorem 4.5 by taking $w_{z}=\left[\widetilde{\omega}_{\mu}\right]$ for every $z \in M^{T}$.

We expect that Theorem 4.8, together with the flexibility of the choice of $d_{i}$ 's in Proposition 4.4, may provide a more simple formula of Proposition 4.4. Indeed, the coefficients and the determinant of $A_{l}(M, \omega)$ can be expressed by very simple formulas in the following special case [CK2]. More precisely, the authors proved in [CK2] that if there exists a vector $\xi \in \mathfrak{t}$ such that $\mu_{\xi}(v)=\lambda_{v}$ for each fixed point $v$, i.e., $\mu_{\xi}$ is a self-indexing moment map, then $(M, \omega)$ satisfies the hard Lefschetz property by computing the determinant of $A_{l}(M, \omega)$ for each $l$.

\section{SiX-Dimensional Hamiltonian GKM manifolds With indeX InCREASing GRaPhS}

In this section, we restrict our attention to six-dimensional Hamiltonian GKM manifolds and give the proof of Theorem 1.3.

Let $(M, \omega)$ be a six-dimensional closed symplectic manifold and let $T$ be a two-dimensional compact torus acting on $(M, \omega)$. Assume that the $T$-action is Hamiltonian GKM with a moment map $\mu: M \longrightarrow \mathfrak{t}^{*}$. Let $\xi \in \mathfrak{t}$ be a generic vector having rational slop such that the corresponding GKM graph $\left(\Gamma, V_{\Gamma}, E_{\Gamma}\right)$ is index increasing with respect to $\xi$. Note that the vector $\xi$ defines a circle subgroup $S^{1}$ of $T$ and the induced $S^{1}$-action on $(M, \omega)$ is Hamiltonian with respect to a moment map $\mu_{\xi}=\langle\mu, \xi\rangle$. We start with the following well-known fact.

Lemma 5.1. $[\mathrm{Au}] b_{\text {odd }}(M)=0$.

Proof. See Remark 3.2.

We reformulate Theorem 1.3 by using equivariant Thom classes defined in Section 3. Recall that $(M, \omega)$ satisfies the hard Lefschetz property if and only if the Hodge-Riemann bilinear form $\mathrm{HR}_{l}$ is nondegenerate for every $l=0, \cdots, 3$, see Section 1 . It is straightforward that

$$
\begin{aligned}
& \mathrm{HR}_{0}: H^{0}(M) \times H^{0}(M) \longrightarrow \mathbb{R} \\
& (\alpha, \beta) \quad \longmapsto<\alpha \beta[\omega]^{3},[M]>
\end{aligned}
$$

is nondegenerate since $\omega^{3}$ is a volume form on $M$. Therefore, by Lemma $5.1, \mathrm{HR}_{2}$ is non-degenerate if and only if $(M, \omega)$ satisfies the hard Lefschetz property. 
Now, let $\tau_{v}^{+}$and $\tau_{v}^{-}$be the equivariant Thom classes for each vertex $v \in V_{\Gamma}$ with respect to $\xi$ and $-\xi$, respectively. Let $b_{2}:=b_{2}(M)$ be the second Betti number of $M$ and let

$$
\left\{p_{1}, \cdots, p_{b_{2}}\right\} \quad \text { and } \quad\left\{q_{1}, \cdots, q_{b_{2}}\right\}
$$

be the sets of index-two vertices and index-four vertices, respectively. These sets have the same number of elements by the Poincaré duality. Let $x_{1}$ and $x_{2}$ be the generators of $S\left(\mathfrak{t}^{*}\right) \cong H^{*}(B T)=\mathbb{R}\left[x_{1}, x_{2}\right]$ given in (2.1). By Theorem 3.9, we may identify $H_{T}^{*}(M)$ with $H(\Gamma, \alpha)$ and the set of all equivariant Thom classes forms a basis of $H_{T}^{*}(M)$ as an $H^{*}(B T)$-module by Theorem 3.8. In particular, each of

$$
\left\{x_{1}, x_{2}, \tau_{p_{k}}^{+} \mid 1 \leq k \leq b_{2}\right\} \quad \text { and } \quad\left\{x_{1}, x_{2}, \tau_{q_{j}}^{-} \mid 1 \leq j \leq b_{2}\right\}
$$

becomes a basis of $H_{T}^{2}(M)$ as an $\mathbb{R}$-vector space.

Lemma 5.2. Let $f: M \hookrightarrow M \times_{T}$ ET be an inclusion of a fiber $M$ given in (2.2) and let $f^{*}: H_{T}^{*}(M) \rightarrow$ $H^{*}(M)$ be its induced ring homomorphism. Then $\mathrm{HR}_{2}$ is nondegenerate if and only if the $b_{2} \times b_{2}$ matrix

is nonsingular.

$$
A_{2}(M, \omega)=\left(a_{j k}\right)_{1 \leq j, k \leq b_{2}}:=\left(\operatorname{HR}_{2}\left(f^{*} \tau_{p_{k}}^{+}, f^{*} \tau_{q_{j}}^{-}\right)\right)_{1 \leq j, k \leq b_{2}}
$$

Proof. Recall that $\mathcal{B}_{2}^{+}=\left\{f^{*} \tau_{p_{1}}^{+}, \cdots, f^{*} \tau_{p_{b_{2}}}^{+}\right\}$and $\mathcal{B}_{2}^{-}=\left\{f^{*} \tau_{q_{1}}^{-}, \cdots, f^{*} \tau_{q_{b_{2}}}^{-}\right\}$are bases of $H^{2}(M)$ by Theorem 3.8 and Theorem 2.3. Then $A_{2}(M, \omega)$ is the matrix representing $\mathrm{HR}_{2}$ with respect to the pair $\left(\mathcal{B}_{2}^{+}, \mathcal{B}_{2}^{-}\right)$and this finishes the proof.

Using Lemma 5.2, we can reformulate Theorem 1.3 into the following proposition.

Proposition 5.3 (Theorem 1.3). The matrix $A_{2}(M, \omega)$ is nonsingular.

Let $o$ (resp. $r$ ) be the unique index-zero (resp. index-six) vertex of $\Gamma$. Recall that vol and $\Theta$ are functions on the edge set $E_{\Gamma}$ defined by

$$
\operatorname{vol}(p, q):=(\mu(q)-\mu(p)) / \alpha(p, q) \in Q\left(\mathfrak{t}^{*}\right)
$$

and

$$
\Theta(p, q):=\frac{\rho_{\alpha(p, q)}\left(\Lambda_{p}^{+}\right)}{\rho_{\alpha(p, q)}\left(\Lambda_{q}^{+} / \alpha(q, p)\right)} \in Q\left(\mathfrak{t}^{*}\right)
$$

for $(p, q) \in E_{\Gamma}$. See Section 4.1. The following proposition is straightforward by Corollary 4.7.

Proposition 5.4. Let $A_{2}(M, \omega)=\left(a_{j k}\right)_{1 \leq j, k \leq b_{2}}$ be given in Lemma 5.2. Then

(1) $a_{j k}=\Theta\left(p_{k}, q_{j}\right) \cdot \operatorname{vol}\left(p_{k}, q_{j}\right)$, and

(2) $a_{j k}$ is nonzero if and only if $p_{k}$ and $q_{j}$ are adjacent.

Suppose that $(p, q) \in E_{\Gamma}$ where $p$ (resp. $q$ ) is an index-two (resp. index-four) vertex of $\Gamma$. Then there exists a unique vertex $v \neq p$ adjacent to and below $q$ with respect to $\xi$, that is, $\mu_{\xi}(v)<\mu_{\xi}(q)$. Also, by the index increasing property, the index of $v$ is less than or equal to two. Since

$$
\text { supp } \tau_{p}^{+} \subset\{p\} \cup\{\text { index- } 4 \text { vertices adjacent to } p\} \cup\{\text { the index- } 6 \text { vertex } r\}
$$

by Theorem 3.8, we have $\tau_{p}^{+}(v)=0$, and therefore $\tau_{p}^{+}(q)=k \cdot \alpha(q, v)$ for some real number $k \in \mathbb{R}$ by Lemma 3.7.(1). Furthermore, since $\tau_{p}^{+}(q)-\tau_{p}^{+}(p) \equiv 0$ modulo $\alpha(p, q)$, we can easily see that $k=\Theta(p, q)$, and therefore

$$
\tau_{p}^{+}(q)=\Theta(p, q) \cdot \alpha(q, v)
$$

see Figure 4.1 and Figure 5.1.(a). Note that if $p$ and $q$ are not adjacent, then $\tau_{p}^{+}(q)=0$ by (5.1). Thus we obtain the following lemma. See also [GT, Theorem 4.1].

Lemma 5.5. Let $p$ and $q$ be an index-two and index-four vertices, respectively. Then $p$ and $q$ are adjacent if and only if $\tau_{p}^{+}(q) \neq 0$. 


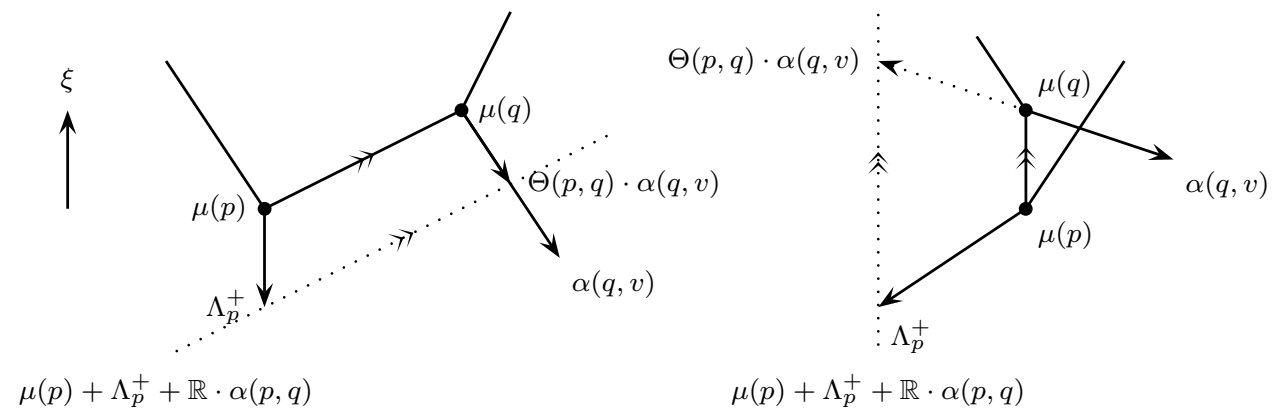

(a)

(b)

Figure 5.1. (a) $\Theta(p, q)>0$, (b) $\Theta(p, q)<0$

5.1. Positivity of $\Theta$. The positivity of $\Theta(p, q)$ will play an essential role for proving the non-singularity of $A_{2}(M, \omega)$.

Definition 5.6. A subset of a real two-dimensional vector space $V$ is said to be in the same side with respect to a straight line $L$ in $V$ if it is contained in the closure of a connected component of $V-L$.

Let $(p, q) \in E_{\Gamma}$ for an index-two vertex $p$ and an index-four vertex $q$, respectively. By definition of graph cohomology, we have

$$
\tau_{p}^{+}(p) \equiv \tau_{p}^{+}(q) \bmod \alpha(p, q)
$$

Substituting

$$
\tau_{p}^{+}(p)=\Lambda_{p}^{+} \quad \text { and } \quad \tau_{p}^{+}(q)=\Theta(p, q) \cdot \alpha(q, v)
$$

into (5.3), we have

$$
-\Lambda_{p}^{+}+\Theta(p, q) \cdot \alpha(q, v)=k \cdot \alpha(p, q) \quad \text { for some real number } k .
$$

Adding $\mu(q)-\mu(p)=\operatorname{vol}(p, q) \cdot \alpha(p, q)$ to both sides of (5.4), we have

$$
\left(-\mu(p)-\Lambda_{p}^{+}\right)+(\mu(q)+\Theta(p, q) \cdot \alpha(q, v))=k^{\prime} \cdot \alpha(p, q) \quad \text { where } k^{\prime}=k+\operatorname{vol}(p, q) .
$$

This implies that

$$
\mu(q)+\Theta(p, q) \cdot \alpha(q, v) \in\left(\mu(p)+\Lambda_{p}^{+}\right)+\mathbb{R} \cdot \alpha(p, q),
$$

that is, $\mu(q)+\Theta(p, q) \cdot \alpha(q, v)$ is contained in the dotted line in Figure 5.1.

On the other hand, $\Theta$ can be understood in the following way : the straight line $\mu(q)+\mathbb{R} \cdot \alpha(q, v)$ intersects $\mu(p)+\Lambda_{p}^{+}+\mathbb{R} \cdot \alpha(p, q)$ at $\mu(q)+\Theta(p, q) \cdot \alpha(q, v)$, see Figure 5.1 in which the line segment connecting $\mu(p)$ and $\mu(q)$ is parallel with the dotted straight line marked by the doubled arrow vector. Consequently, we can see that $\mu(p)+\Lambda_{p}^{+}$and $\mu(q)+\Theta(p, q) \cdot \alpha(q, v)$ are in the same side with respect to the straight line $\mu(p)+\mathbb{R} \cdot \alpha(p, q)$. Equivalently, $\Lambda_{p}^{+}$and $\Theta(p, q) \cdot \alpha(q, v)$ are in the same side with respect to the straight line $\mathbb{R} \cdot \alpha(p, q)$. From this observation, we deduce the following.

Lemma 5.7. Two vectors $\Lambda_{p}^{+}$and $\alpha(q, v)$ are in the same side with respect to the straight line $\mathbb{R} \cdot \alpha(p, q)$ if and only if $\Theta(p, q)$ is positive.

As the following examples show, Lemma 5.7 enables us to check the positivity of $\Theta$ by looking up the shape of a graph.

Example 5.8. For example, $\Theta(p, q)$ is positive in Figure 5.1.(a). On the other hand, in Figure 5.1.(b), $\Theta(p, q)$ is negative since two vectors $\Lambda_{p}^{+}$and $\alpha(q, v)$ are not in the same side with respect to the straight line $\mathbb{R} \cdot \alpha(p, q)$. 
More concrete examples are as follows. In Figure 5.4.(d), $\Theta(p, q)$ is negative for the index-two vertex $p$ and the index-four vertex $q$ which lie on the interior of the moment map image. In fact, Figure 5.4.(d) corresponds to Tolman's example of a non-Kähler Hamiltonian GKM manifold explained in Example 1.4. On the contrary, $\Theta(p, q)$ is positive for any $(p, q) \in E_{\Gamma}$ in Figure 5.4 with ind $p=2$ and ind $q=4$.

By using Lemma 5.7, we can state a more refined condition under which $\Theta(p, q)$ is positive. For an index-two vertex $p$, we denote by $\gamma_{p}$ the cycle whose vertices consist of $p$ and vertices connected by ascending paths starting at $p$, and call it the ascending cycle starting at $p$. In other words, the set of all vertices contained in $\gamma_{p}$ is the right hand side of (5.1). Note that $p$ is of index-two so that $p$ should be adjacent to at least one and at most two index-four vertices by the three valency of $\Gamma$, see Section 3.1. In particular, $\gamma_{p}$ has three or four vertices. An ascending cycle is called triangular (resp. tetragonal) if it has three (resp. four) vertices. In other words, $\gamma_{p}$ is triangular if and only if $p$ is adjacent to exactly one index-four vertex and to $r$. Also, $\gamma_{p}$ is tetragonal if and only if $p$ is adjacent to exactly two index-four vertices.

Example 5.9. Let us consider examples of ascending cycles in Figure 5.4. Each of (a) and (b) has one triangular and no tetragonal ascending cycle. And each of (c), (e), and (f) has one triangular and one tetragonal ascending cycles. Each of (d) and (g) has no triangular ascending cycle and it has two tetragonal ascending cycles. And (h) has no triangular ascending cycle and has three tetragonal ascending cycles.

For a tetragonal ascending cycle $\gamma_{p}$ starting at $p$, we denote by $\square \gamma_{p}$ the union of images $\mu\left(S_{e}^{2}\right)$ for edges $e$ of $\gamma_{p}$. Thus $\square \gamma_{p}$ is a tetragon in $\mathfrak{t}^{*}$. It is classical that tetragons are classified into three types as follows, see Figure 5.2.

Lemma 5.10. [We, p.50] Tetragons $\square A B C D$ in a plane are classified into three types :

(a) convex if for each edge $\ell$ of $\square A B C D,\{A, B, C, D\}$ is in the same side with respect to the straight line generated by $\ell$,

(b) concave if the convex hull $\operatorname{Conv}\{A, B, C, D\}$ is triangular, i.e., a vertex is contained in the interior of $\operatorname{Conv}\{A, B, C, D\}$,

(c) crossed if there exist two opposite line segments passing through each other.

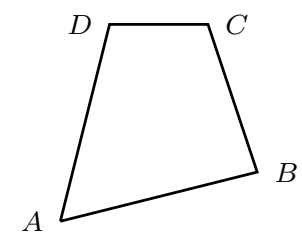

(a) convex

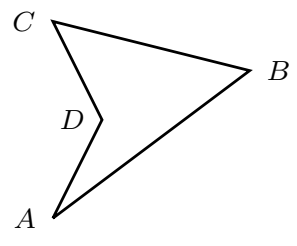

(b) concave

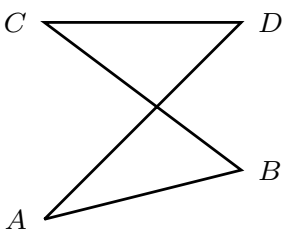

(c) crossed

FiguRE 5.2. Three types of tetragons

Similarly, we call a tetragonal ascending cycle $\gamma_{p}$ convex, concave, or crossed if the tetragon $\square \gamma_{p}$ is convex, concave, or crossed, respectively. We introduce a new condition which guarantees that $\Theta(p, q)$ is positive.

Proposition 5.11. For an adjacent index-two vertex $p$ and an index-four vertex $q$, if the ascending cycle $\gamma_{p}$ is tetragonal and convex, then $\Theta(p, q)$ is positive.

Before we prove Proposition 5.11, we give the following lemma without proof, which is essentially the same as Lemma 3.10.

Lemma 5.12. For each oriented edge $e \in E_{\Gamma}$, we can reorder $\alpha_{j, i(e)}$ 's and $\alpha_{j, t(e)}$ 's so that (1) $\alpha_{n, t(e)}=$ $-\alpha_{n, i(e)}=-\alpha(e)$, and (2) $\alpha_{j, t(e)}, \alpha_{j, i(e)}$ are in the same side with respect to $\mathbb{R} \cdot \alpha(e)$ for each $1 \leq j \leq n-1$. 
Proof of Proposition 5.11. Pick the vertex $v \neq p$ which is adjacent to and below $q$. By the assumption, there exists another index-four vertex $q^{\prime} \neq q$ which is adjacent to and above $p$, see Figure 5.3. Since $\square \gamma_{p}$ is convex, $\alpha\left(p, q^{\prime}\right)$ and $\alpha(q, r)$ are in the same side with respect to $\mathbb{R} \cdot \alpha(p, q)$ by Lemma 5.10. Applying Lemma 5.12 to the edge $(p, q)$, two weights $\Lambda_{p}^{+}=\alpha(p, o)$ and $\alpha(q, v)$ should be in the same side with respect to $\mathbb{R} \cdot \alpha(p, q)$. Therefore, $\Theta(p, q)$ is positive by Lemma 5.7 .

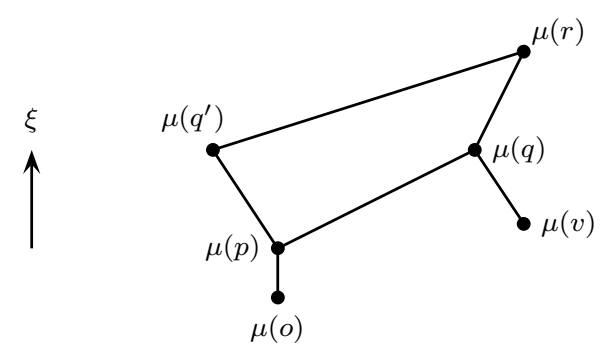

FiguRE 5.3. Proof of Proposition 5.11

Example 5.13. Let us consider Figure 5.4.(d). For the index-two vertex $p$ in the interior of $\mu(M), \gamma_{p}$ is not convex but concave. As we have seen in Example 5.8, $\Theta(p, q)$ is negative for the index-four vertex $q$ in the interior of $\mu(M)$. On the other hand, for any other index-two vertex $p$ in Figure 5.4, if $\gamma_{p}$ is tetragonal, it is convex.

5.2. Weak classification. In addition to Proposition 5.4 and Proposition 5.11, we need to understand the GKM graph more precisely to show that the determinant of the matrix $A_{2}(M, \omega)$ is nonzero. Let $\mathcal{E}$ and $\mathcal{V}$ be the numbers of non-oriented edges and vertices of $\Gamma$, respectively.

Lemma 5.14. Let $\mathcal{V}$ and $\mathcal{E}$ be given above. Then

- $2 \mathcal{E}=3 \mathcal{V}$, and

- the number of index-two vertices, i.e., $b_{2}$ is equal to $\mathcal{V} / 2-1$.

Proof. The first statement follows from the three valency of $\Gamma$. Also, the second statement follows from the Poincaré duality.

The following proposition classifies all possible GKM graphs $\Gamma$ into eight types according to the following four criteria :

(1) the shape of the moment map image $\mu(M)$,

(2) the number of vertices of $\Gamma$,

(3) adjacency between $o$ and $r$, and

(4) the number of tetragonal ascending cycles starting at index-two vertices.

Proposition 5.15. Let $(M, \omega)$ be a six-dimensional closed Hamiltonian $T^{2}$-manifold. Suppose that the action is GKM and its GKM graph $\Gamma$ is index-increasing with respect to some generic $\xi \in \mathfrak{t}$. Then $\Gamma$ satisfies one of $(a) \sim(h)$ of Table 5.1 .

The proof of Proposition 5.15 will be given in Section 6. In Figure 5.4, examples of the eight types of GKM graphs in Table 5.1 are illustrated. Note that Proposition 5.15 does not claim that every possible index increasing GKM graph is one of those in Figure 5.4. For example, there exists an index increasing GKM graph satisfying Table 5.1.(h) but is different from Figure 5.4.(h), see Figure 5.5. Thus we may call Proposition 5.15 a weak classification of index increasing GKM graphs of closed six-dimensional Hamiltonian GKM manifolds. Nevertheless, Table 5.1.(a) $(\mathrm{g})(\mathcal{V} \leq 6)$ are corresponding to the Morton's classification of index increasing GKM graphs of closed six-dimensional Hamiltonian GKM manifolds with vertices less than or equal to six, see [Mo]. 


\begin{tabular}{c||c|c|c|c} 
& $\mu(M)$ & $\mathcal{V}$ & o is adjacent to $r ?$ & $\begin{array}{c}\text { the number of tetragonal ascending cycles } \\
\text { starting at an index-two vertex }\end{array}$ \\
\hline \hline (a) & triangle & 4 & Yes & 0 \\
(b) & tetragon & 4 & Yes & 0 \\
(c) & tetragon & 6 & No & 1 \\
(d) & tetragon & 6 & Yes & 2 \\
(e) & pentagon & 6 & No & 1 \\
(f) & hexagon & 6 & No & 1 \\
(g) & hexagon & 6 & Yes & 2 \\
(h) & hexagon & 8 & No & 3
\end{tabular}

TABLE 5.1. Eight types of possible index increasing GKM graphs

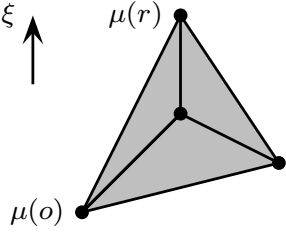

(a)

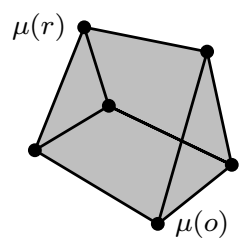

(e)

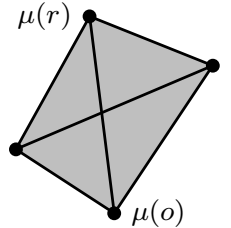

(b)

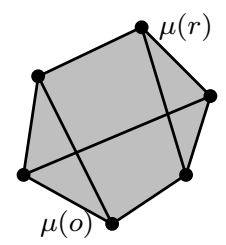

(f)

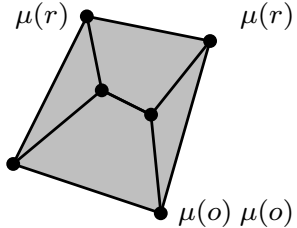

(c)

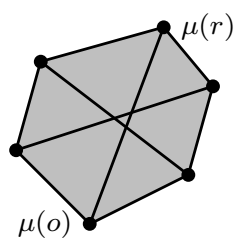

(g)

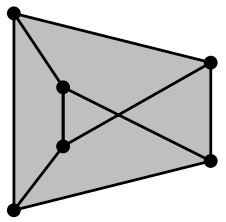

(d)

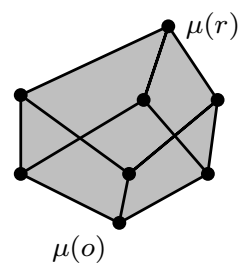

(h)

FiguRE 5.4. Examples of eight types of possible index increasing GKM graphs
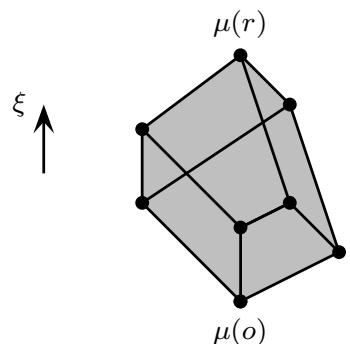

Figure 5.5. Example of Table 5.1.(h)

Remark 5.16. We can easily check that Tolman's example (Example 1.4) corresponds to Table 5.1.(d). See also [GT, Example 5.2 and Figure 1].

The following proposition will be used to prove our main theorem in Section 5.3, where the proof will be given in Section 6 .

Proposition 5.17. Suppose that $\Gamma$ is of type Table 5.1.(h). Then every tetragonal ascending cycle in $\Gamma$ is convex. In particular, $\Theta(p, q)$ is positive for every index-two vertex $p$ and index-four vertex $q$ of $\Gamma$.

5.3. Proof of Theorem 1.3. Now, we are ready to prove our main theorem.

Proof of Proposition 5.3. (Proof of Theorem 1.3) We first consider the case where a GKM graph $\Gamma$ satisfies Table 5.1.(a) or (b). In this case, we have $b_{2}=1$ and $H^{2}(M)$ is generated by the symplectic class [ $\left.\omega\right]$. Since $\left[\omega^{2}\right] \neq 0$ in $H^{4}(M)$, the hard Lefschetz property of $(M, \omega)$ is automatically satisfied. 
Second, suppose that $\Gamma$ satisfies Table 5.1.(c), (e), or (f). In this case, we have $b_{2}=2$ and there is only one tetragonal ascending cycle starting at an index-two vertex. (See Table 5.1.) This implies that the number of non-oriented edges connecting an index-two vertex and a four vertex is three. Since $A_{2}(M, \omega)$ is a $2 \times 2$ matrix with exactly three nonzero entries by Proposition $5.4, A_{2}(M, \omega)$ should be nonsingular.

Third, assume that $\Gamma$ satisfies Table 5.1.(h). Then $\Gamma$ has three index-two vertices so that $b_{2}=3$. In particular, $A_{2}(M, \omega)$ is a $3 \times 3$ matrix. Also, there are three tetragonal ascending cycles starting at an index-two vertex, that is, the ascending cycle starting at an index-two vertex $p_{k}$ is tetragonal for every $k=1,2,3$. Moreover, the ascending cycles are convex by Proposition 5.17. Thus if $p_{k}$ and $q_{j}$ are adjacent, then $\Theta\left(p_{k}, q_{j}\right)$ is positive by Proposition 5.11, and therefore $a_{j k}$ is positive for $1 \leq j, k \leq 3$ with $\left(p_{k}, q_{j}\right) \in E_{\Gamma}$ by Proposition 5.4. So, by Proposition 5.4, there are exactly three zeros in $A_{2}(M, \omega)$ and each zero appears exactly one time in each row and column. Reordering $p_{k}$ 's and $q_{j}$ 's, if necessary, we may assume that the diagonal entries of $A_{2}(M, \omega)$ are all zero. Then,

$$
\operatorname{det} A_{2}(M, \omega)=a_{12} a_{23} a_{31}+a_{13} a_{21} a_{32}>0 .
$$

Therefore, $A_{2}(M, \omega)$ is nonsingular.

Finally, consider the case where $\Gamma$ satisfies Table 5.1.(d) or (g). In this case, we have $b_{2}=2$ (and hence $A_{2}(M, \omega)$ is a $2 \times 2$ matrix) and there are two tetragonal ascending cycles starting at an index-two vertex. In other words, the ascending cycle starting at each index-two vertex $p_{k}$ is tetragonal and hence it is adjacent to both $q_{1}$ and $q_{2}$. Thus all entries of $A_{2}(M, \omega)$ are nonzero by Proposition 5.4.

To show that the determinant of $A_{2}(M, \omega)$ is nonzero, we apply column operation on $A_{2}(M, \omega)$ to obtain a triangular matrix. To do this, we need the following lemma.

Lemma 5.18. Let $t_{1}$ and $t_{2}$ be two arbitrary nonzero real numbers. If $\Gamma$ satisfies Table 5.1.(d) or $(g)$, then the following (degree four) graph cohomology class

$$
t_{1} \cdot \tau_{p_{1}}^{+} \cdot\left(\left[\widetilde{\omega_{\mu}}\right]+\mu\left(p_{1}\right)\right)+t_{2} \cdot \tau_{p_{2}}^{+} \cdot\left(\left[\widetilde{\omega_{\mu}}\right]+\mu\left(p_{2}\right)\right)
$$

does not vanish simultaneously on $q_{1}$ and $q_{2}$.

Proof. Note that the class (5.5) vanishes on $o, p_{1}, p_{2}$ by Theorem 3.8 and Lemma 2.7. Moreover, if (5.5) vanishes on $q_{1}$ and $q_{2}$ simultaneously, then (5.5) should be the zero class by Lemma 3.7.(2). Thus we need only show that (5.5) never vanishes on $r$.

Therefore, it is enough to prove that the following two linear polynomials

$$
\left[\tau_{p_{1}}^{+} \cdot\left(\left[\widetilde{\omega_{\mu}}\right]+\mu\left(p_{1}\right)\right)\right](r) \text { and }\left[\tau_{p_{2}}^{+} \cdot\left(\left[\widetilde{\omega_{\mu}}\right]+\mu\left(p_{2}\right)\right)\right](r)
$$

in $\mathrm{S}\left(\mathfrak{t}^{*}\right)$ are $\mathbb{R}$-linearly independent. We first compute $\tau_{p_{k}}^{+}(r)$ as follows. Since $\tau_{p_{k}}^{+}$is zero at $o$ for $k=1,2$ by Theorem 3.8, and $o$ and $r$ are adjacent by Table 5.1.(d) and (g), we have

$$
\tau_{p_{k}}^{+}(r)=d_{k} \cdot \alpha(r, o)
$$

for some real numbers $d_{k}$ by Lemma 3.7.(1).

We claim that $d_{k}$ 's are all nonzero. Suppose that $d_{k}$ is zero for some $k$, i.e. $\tau_{p_{k}}^{+}(r)=0$. Without loss of generality, we may assume that $k=1$. Then $\tau_{p_{1}}^{+}$vanishes on $r$. Moreover, $\tau_{p_{1}}^{+}$vanishes on $p_{2}$ by (5.1). Since each of $q_{1}$ and $q_{2}$ is adjacent to both $p_{1}$ and $p_{2}, \tau_{p_{1}}^{+}\left(q_{j}\right)$ is divided by both $\alpha\left(q_{j}, r\right)$ and $\alpha\left(q_{j}, p_{2}\right)$ for each $j=1,2$ by Lemma 3.7.(1). However, two weights $\alpha\left(q_{j}, r\right)$ and $\alpha\left(q_{j}, p_{2}\right)$ are linearly independent by the GKM condition (2) and $\tau_{p_{1}}^{+}\left(q_{j}\right)$ is of polynomial degree 1 in $\mathrm{S}\left(\mathrm{t}^{*}\right)$. Thus we have $\tau_{p_{1}}^{+}\left(q_{j}\right)=0$ and it is a contradiction by Lemme 5.5. Thus $d_{1}$ is nonzero. Also, we obtain $d_{2} \neq 0$ in a similar way. Therefore, the polynomials in (5.6) can be expressed by

$$
\left[\tau_{p_{k}}^{+} \cdot\left(\left[\widetilde{\omega_{\mu}}\right]+\mu\left(p_{k}\right)\right)\right](r)=d_{k} \cdot \alpha(r, o) \cdot\left(-\mu(r)+\mu\left(p_{k}\right)\right)
$$

by Lemma 2.7 for $k=1,2$.

Now, it is enough to show that

$$
\mu(r)-\mu\left(p_{1}\right) \quad \text { and } \quad \mu(r)-\mu\left(p_{2}\right)
$$


are $\mathbb{R}$-linearly independent. To the contrary, suppose that they are linearly dependent. Then $\mu(r), \mu\left(p_{1}\right)$, and $\mu\left(p_{2}\right)$ should be collinear. Let us first consider the case of Table 5.1.(d). Then there exists indexfour interior vertex, which is assumed to be $q_{1}$, adjacent to $r, p_{1}$, and $p_{2}$. Similarly, we can easily see that $r$ is adjacent to $o, q_{1}$, and $q_{2}$. Note that if $\mu\left(p_{1}\right)$ and $\mu\left(p_{2}\right)$ are in the same side with respect to the straight line $\overleftrightarrow{\mu(r) \mu\left(q_{1}\right)}$, then both $\mu(o)$ and $\mu\left(q_{2}\right)$ must be in the same side with $\mu\left(p_{k}\right)$ 's by Lemma 5.12 so that $\overleftrightarrow{\mu(r) \mu\left(q_{1}\right)}$ is on the boundary of $\mu(M)$, which contradicts that $q_{1}$ is an interior point of the moment polytope $\mu(M)$. Thus $\mu\left(p_{1}\right)$ and $\mu\left(p_{2}\right)$ cannot be in the same side with respect to the straight line $\overleftrightarrow{\mu(r) \mu(q)}$, and hence $\mu(r), \mu\left(p_{1}\right)$, and $\mu\left(p_{2}\right)$ are not colinear.

For the case of $(\mathrm{g}), \mu\left(p_{1}\right)$ and $\mu\left(p_{2}\right)$ are vertices of the moment polytope $\mu(M)$ as well as $\mu(r)$. Then it is straightforward that $\mu(r)-\mu\left(p_{1}\right)$ and $\mu(r)-\mu\left(p_{2}\right)$ are linearly independent.

We go back to the proof of Proposition 5.3. Since every $a_{j k}$ is nonzero, we can take $t_{0}=-\frac{a_{12}}{a_{11}} \neq 0$ so that $a_{12}+t_{0} \cdot a_{11}=0$. Since

$$
\operatorname{det}\left(\begin{array}{ll}
a_{11} & a_{12} \\
a_{21} & a_{22}
\end{array}\right)=\operatorname{det}\left(\begin{array}{cc}
a_{11} & a_{12}+t_{0} \cdot a_{11} \\
a_{21} & a_{22}+t_{0} \cdot a_{21}
\end{array}\right)=\operatorname{det}\left(\begin{array}{cc}
a_{11} & 0 \\
a_{21} & a_{22}+t_{0} \cdot a_{21}
\end{array}\right),
$$

it is enough to show that $a_{22}+t_{0} \cdot a_{21} \neq 0$.

Consider the following equivariant cohomology class

$$
\tau_{p_{k}}^{+} \cdot\left(\left[\widetilde{\omega_{\mu}}\right]+\mu\left(p_{k}\right)\right) \cdot \tau_{q_{j}}^{-} \in H_{T}^{6}(M)
$$

Using $f^{*}\left(\left[\widetilde{\omega_{\mu}}\right]-\mu\left(p_{k}\right)\right)=[\omega]$ and the ABBV-localization theorem, we have

$$
\begin{aligned}
a_{j k} & =\left\langle f^{*}\left(\tau_{p_{k}}^{+}\right) \wedge[\omega] \wedge f^{*}\left(\tau_{q_{j}}^{-}\right),[M]\right\rangle \\
& =\int_{M} \tau_{p_{k}}^{+} \cdot\left(\left[\widetilde{\omega}_{\mu}\right]+\mu\left(p_{k}\right)\right) \cdot \tau_{q_{j}}^{-} \\
& =\sum_{v \in V_{\Gamma}}\left[\tau_{p_{k}}^{+} \cdot\left(\left[\widetilde{\omega}_{\mu}\right]+\mu\left(p_{k}\right)\right) \cdot \tau_{q_{j}}^{-}\right](v) / \Lambda_{v} \\
& =\left[\tau_{p_{k}}^{+} \cdot\left(\left[\widetilde{\omega}_{\mu}\right]+\mu\left(p_{k}\right)\right) \cdot \tau_{q_{j}}^{-}\right]\left(q_{j}\right) / \Lambda_{q_{j}} \\
& =\tau_{p_{k}}^{+}\left(q_{j}\right) \cdot\left(-\mu\left(q_{j}\right)+\mu\left(p_{k}\right)\right) / \Lambda_{q_{j}}^{+} \quad\left(=\left[\tau_{p_{k}}^{+} \cdot\left(\left[\widetilde{\omega}_{\mu}\right]+\mu\left(p_{k}\right)\right)\right]\left(q_{j}\right) / \Lambda_{q_{j}}^{+}\right) .
\end{aligned}
$$

In the fourth equality, we use Theorem 3.8 and the followings :

- $\operatorname{supp} \tau_{p_{k}}^{+} \subset\left\{p_{k}\right\} \cup\left\{\right.$ index-4 vertices adjacent to $\left.p_{k}\right\} \cup\{$ the index-6 vertex $r\}$,

- supp $\tau_{q_{j}}^{-} \subset\left\{q_{j}\right\} \cup\left\{\right.$ index-2 vertices adjacent to $\left.q_{j}\right\} \cup\{$ the index-0 vertex $o\}$, and

- $\left(\left[\widetilde{\omega_{\mu}}\right]+\mu\left(p_{k}\right)\right)\left(p_{k}\right)=-\mu\left(p_{k}\right)+\mu\left(p_{k}\right)=0$

obtained from (5.1) and Lemma 2.7. Then, by (5.7), we can easily see that

$$
\begin{aligned}
a_{j k} & =\left[\tau_{p_{k}}^{+} \cdot\left(\left[\widetilde{\omega}_{\mu}\right]+\mu\left(p_{k}\right)\right)\right]\left(q_{j}\right) / \Lambda_{q_{j}}^{+}, \quad \text { and } \\
a_{j 2}+t_{0} \cdot a_{j 1} & =\left[\tau_{p_{2}}^{+} \cdot\left(\left[\widetilde{\omega}_{\mu}\right]+\mu\left(p_{2}\right)\right)+t_{0} \cdot \tau_{p_{1}}^{+} \cdot\left(\left[\widetilde{\omega}_{\mu}\right]+\mu\left(p_{1}\right)\right)\right]\left(q_{j}\right) / \Lambda_{q_{j}}^{+} .
\end{aligned}
$$

Since $t_{0} \neq 0$, both $a_{12}+t_{0} \cdot a_{11}$ and $a_{22}+t_{0} \cdot a_{21}$ do not vanish simultaneously by Lemma 5.18. Therefore, we have $a_{22}+t_{0} \cdot a_{21} \neq 0$. This completes the proof.

\section{Proof of Proposition 5.15 And 5.17}

In this section, we prove Proposition 5.15 and 5.17 used in Section 5. To begin with, we introduce the following terminologies. 
Definition 6.1. A vertex $v$ is called a boundary vertex (resp. an interior vertex) if $\mu(v)$ is contained in the boundary (resp. interior) of $\mu(M)$. Similarly, an edge $e$ is called a boundary edge (resp. an interior edge) if $\mu\left(S_{e}^{2}\right)$ is contained in the boundary (resp. interior) of $\mu(M)$. A path $\left(v_{0}, \cdots, v_{l}\right)$ of $\Gamma$ is called a boundary path if each edge $\left(v_{j}, v_{j+1}\right)$ is a boundary for every $0 \leq j \leq l-1$.

Now, we give the proof of Proposition 5.15 as follows.

Proof of Proposition 5.15. Consider two ascending boundary paths

$$
\left(v_{0}, \cdots, v_{l}\right) \quad \text { and } \quad\left(v_{0}^{\prime}, \cdots, v_{l^{\prime}}^{\prime}\right)
$$

from $o$ to $r$. Then $\mu(M)$ is a convex $\left(l+l^{\prime}\right)$-gon by the Atiyah-Guillemin-Sternberg convexity theorem [At, GS] so that both paths cannot have length one simultaneously. Moreover, by the index increasing property, the lengths of the two paths are less than or equal to three, i.e. $l, l^{\prime} \leq 3$. Therefore, we have

$$
2 \leq l \cdot l^{\prime} \quad \text { and } \quad l, l^{\prime} \leq 3 .
$$

Without loss of generality, we may assume that $l \leq l^{\prime}$. Then there are exactly five possible cases :

$$
\left(l, l^{\prime}\right) \in\{(1,2),(2,2),(1,3),(2,3),(3,3)\} .
$$

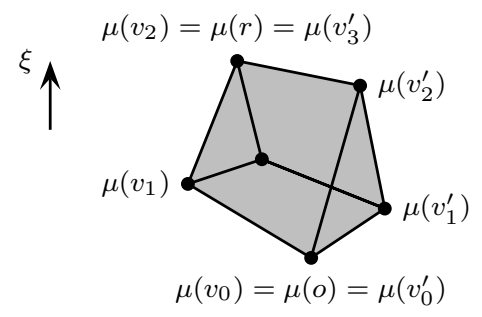

FiguRE 6.1. Example of ascending boundary paths for $\left(l, l^{\prime}\right)=(2,3)$

If $l=1, l^{\prime}=2$, then $\mu(M)$ is a triangle so that $o$ and $r$ are adjacent. We may assume that $v_{1}^{\prime}$ is of index-two. (If not, then $v_{1}^{\prime}$ is of index-two with respect to $-\xi$ so that we need only take $-\xi$ instead of $\xi$.) Then there exists at least one index-four interior vertex by the Poincare duality. Moreover, there cannot exist more than one index-four vertex by the three valency at $r$, since any index-four vertex is adjacent to $r$ by the index-increasing property and $r$ is already adjacent to two vertices $o$ and $v_{1}^{\prime}$. Therefore, $\Gamma$ has only one index-four vertex so that $\Gamma$ has four vertices, that is, $\Gamma$ is a complete graph and the unique ascending cycle $\gamma_{v_{1}^{\prime}}$ starting at the unique index-two vertex $v_{1}^{\prime}$ is triangular. Thus $\Gamma$ is the case of Table 5.1.(a).

If $l=2, l^{\prime}=2$, then $\mu(M)$ is a tetragon. Then each of $o$ and $r$ is adjacent to the boundary vertices $v_{1}$ and $v_{1}^{\prime}$. We first show that $v_{1}$ and $v_{1}^{\prime}$ have different indices. If $v_{1}$ and $v_{1}^{\prime}$ have the same index, say two, then there should be at least two index-four interior vertices by the Poincaré duality. Thus $r$ should be adjacent to at least four vertices and this contradicts the three valency at $r$. Therefore, $v_{1}$ and $v_{1}^{\prime}$ must have different indices. Assume that $v_{1}$ is of index-two and $v_{1}^{\prime}$ is of index-four. Then $\gamma_{v_{1}}$ is triangular since $v_{1}$ is adjacent to $r$.

Now, there are two possible cases according to adjacency of $o$ and $r$. If $o$ and $r$ are adjacent, then $o$ (resp. $r$ ) is adjacent to the three vertices $r, v_{1}$, and $v_{1}^{\prime}$ (resp. o, $v_{1}$, and $v_{1}^{\prime}$ ) so that there is no other vertex except for $o, r, v_{1}$, and $v_{1}^{\prime}$ since any vertex other than $o, r$ should be adjacent to $o$ or $r$ by the index increasing property. In other words, $\Gamma$ has four vertices and $v_{1}$ must adjacent to $v_{1}^{\prime}$. This is the case of Table 5.1.(b).

Second, assume that $o$ and $r$ are not adjacent. Since each of $o$ and $r$ is adjacent to $v_{1}$ and $v_{1}^{\prime}$, there are exactly two interior vertices by the three valency of $\Gamma$ and the Poincare duality, and therefore $\Gamma$ has six vertices. Since $v_{1}$ and $v_{1}^{\prime}$ have different indices, two interior vertices have different indices by the Poincaré duality. Let $p$ and $q$ be the index-two and index-four interior vertex respectively. Note that $v_{1}$ and $v_{1}^{\prime}$ are 
not adjacent, otherwise $\Gamma$ cannot be three-valent at $p$ and $q$. Therefore, $p$ and $v_{1}^{\prime}$ are adjacent and that $v_{1}$ and $q$ are adjacent by the index increasing property. Also $p$ is adjacent to $q$ by the three valency of $\Gamma$. Consequently, $p$ is adjacent to three vertices $q, v_{1}^{\prime}$, o so that $\gamma_{p}$ is tetragonal. This is the case of Table 5.1.(c).
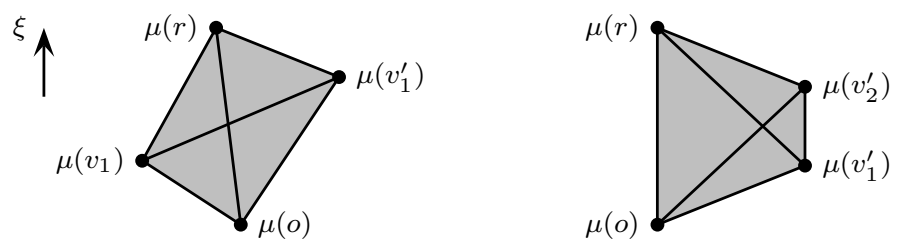

Figure 6.2. Examples of Table 5.1.(b)

If $l=1, l^{\prime}=3$, then $\mu(M)$ is a tetragon and $o$ is adjacent to $r$. Note that $v_{1}^{\prime}$ and $v_{2}^{\prime}$ are of index-two and of index-four by the index increasing property, respectively. Note that $o$ (resp. $r$ ) is adjacent to $r$ and $v_{1}^{\prime}$ (resp. $o$ and $v_{2}^{\prime}$ ) and hence there are at most two interior vertices. By the Poincaré duality, the number of interior vertices is zero or two.

First, if there is no interior vertex, then $\Gamma$ has four vertices and $v_{1}^{\prime}$ (resp. $v_{2}^{\prime}$ ) is adjacent to $r$ (resp. $o$ ), which is the case of Table 5.1.(b). Second, assume that there are two interior vertices, namely, the index-two interior vertex $p$ and the index-four interior vertex $q$. Then $r$ is adjacent to three vertices $o, v_{2}^{\prime}$, and $q$. Similarly, $o$ is adjacent to $r, v_{1}^{\prime}$, and $p$. By the three valency of $\Gamma$, each of $p$ and $v_{1}^{\prime}$ is adjacent to $v_{2}^{\prime}$ and $q$, and therefore the ascending cycles $\gamma_{p}$ and $\gamma_{v_{1}^{\prime}}$ are tetragonal. This is the case of Table 5.1.(d).
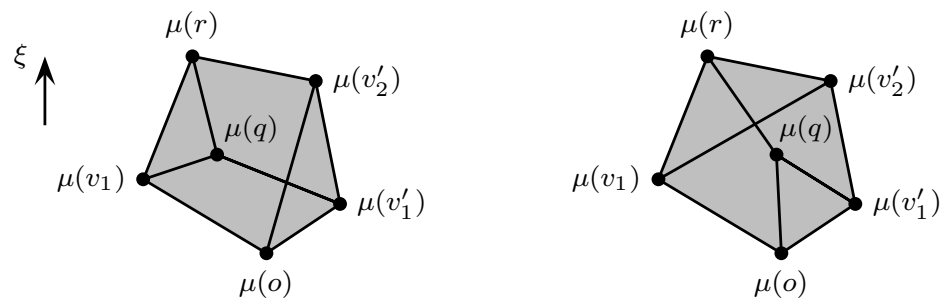

Figure 6.3. The case of $l=2$ and $l^{\prime}=3$

If $l=2, l^{\prime}=3$, then $\mu(M)$ is a pentagon. Since $\left(v_{0}^{\prime}, v_{1}^{\prime}, v_{2}^{\prime}, v_{3}^{\prime}\right)$ is an ascending boundary path from $v_{0}^{\prime}=o$ to $v_{3}^{\prime}=r, v_{1}^{\prime}$ is of index-two and $v_{2}^{\prime}$ is of index-four. Furthermore, we may assume that $v_{1}$ is of index two. Since $v_{1}$ is adjacent to $r$, the ascending cycle $\gamma_{v_{1}}$ is triangular. Note that there is exactly one interior vertex, say $q$, by the three valency of $\Gamma$ and the Poincaré duality. Then $r$ is adjacent to $v_{1}, v_{2}^{\prime}$, and $q$ so that $r$ is not adjacent to $o$. Also, $r$ is not adjacent to $v_{1}^{\prime}$. Thus $v_{1}^{\prime}$ is adjacent to $q$ and $v_{2}^{\prime}$ so that the ascending cycle $\gamma_{v_{1}^{\prime}}$ is tetragonal. Consequently, there is only one tetragonal ascending cycle $\gamma_{v_{1}^{\prime}}$ and this is the case Table 5.1.(e).

If $l=3, l^{\prime}=3$, then $\mu(M)$ is a hexagon. By index increasing property, $v_{1}$ and $v_{1}^{\prime}$ are of index-two, and $v_{2}$ and $v_{2}^{\prime}$ are of index four. By the three valency at $o$ and $r$, there exist at most two interior vertices so that there are two possible cases according to the number of interior vertices. If $\Gamma$ has six vertices (with no interior vertex), then this is the case of Table 5.1.(f) if $o$ and $r$ is not adjacent, and of Table 5.1.(g) if $o$ and $r$ is adjacent. Also, if $\Gamma$ has eight vertices (with two interior vertices), then $r$ should be adjacent to three index-four vertices so that $r$ is not adjacent to $o$. Thus any ascending cycle is tetragonal and this is the case of Table 5.1.(h)

Now, we prove Proposition 5.17. We first recall the following.

Lemma 6.2. A vertex $v$ is an interior vertex if and only if $\sum_{1 \leq j \leq 3} \mathbb{R}^{+} \cdot \alpha_{j, v}=\mathfrak{t}^{*}$. In particular, if $v$ is an interior vertex, then $\alpha_{1, v}, \alpha_{2, v}$ are not in the same side with respect to $\mathbb{R} \cdot \alpha_{3, v}$.

Proof. See [Km, Lemma 2 and Example 2]. 
Proof of Proposition 5.1\%. We label each vertex as in Figure 6.4 :

- two ascending boundary paths from $o$ to $r$ are $\left(o, p_{1}, q_{1}, r\right)$ and $\left(o, p_{3}, q_{3}, r\right)$, and

- $p_{2}$ and $q_{2}$ are the interior vertices of index-two and four, respectively.

Note that

- every ascending cycle starting at an index-two vertex is tetragonal by Table 5.1, and therefore each $p_{k}$ (resp. $q_{j}$ ) is not adjacent to $r$ (resp. o), and

- each tetragonal ascending cycle contains two index-four vertices, it contains at least one boundary vertex of index-four.

We also note that, by interchanging $p_{1}$ and $p_{3}$ (resp. $q_{1}$ and $q_{3}$ ) if necessary, there are exactly four types of ascending cycles in $\Gamma$ : (i) an ascending cycle $\gamma_{p}$ ( $p$ is any index-two vertex) contains $q_{1}$ and $q_{3}$, (ii) $\gamma_{p_{1}}$ contains $q_{1}$ and $q_{2}$, (iii) $\gamma_{p_{2}}$ contains $q_{1}$ and $q_{2}$, and (iv) $\gamma_{p_{3}}$ contains $q_{1}$ and $q_{2}$.

Case (i): $\gamma_{p}$ contains $q_{1}$ and $q_{3}$. Note that $\left(q_{1}, r\right)$ and $\left(q_{3}, r\right)$ of $\gamma_{p}$ are boundary edges so that $\gamma_{p}$ cannot be crossed by Lemma 5.10, see Figure 6.4.(a) for example. Furthermore, $p$ is below $q_{1}, q_{3}$, and $r$ by the index increasing property so that $p$ is not contained in the interior of $\operatorname{Conv}\left\{p, q_{1}, q_{3}, r\right\}$. Thus $\gamma_{p}$ is convex.

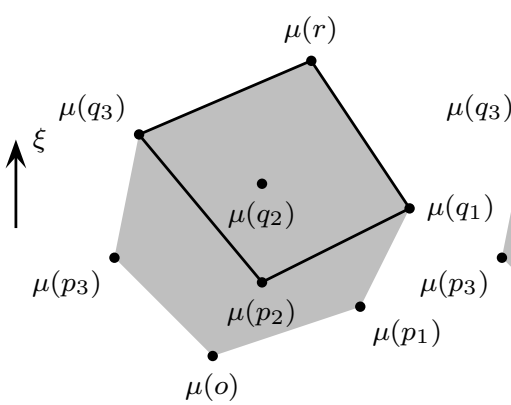

(a)

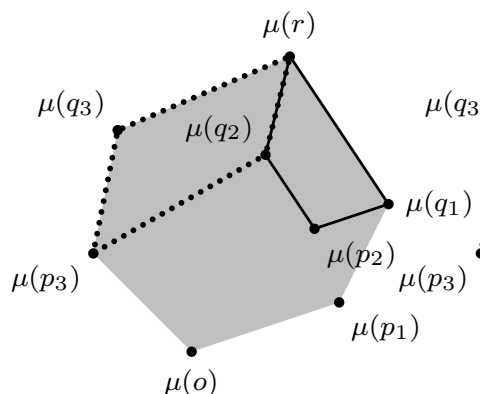

(d)

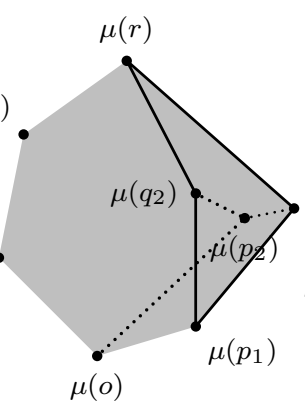

(b)

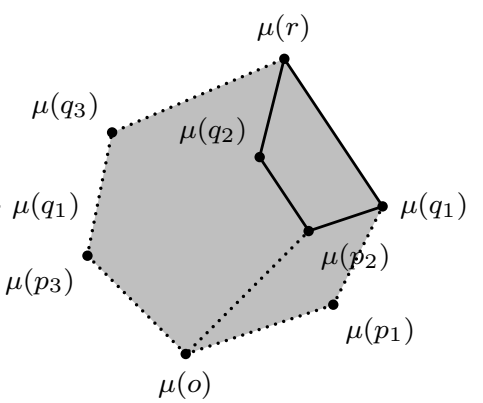

(c)

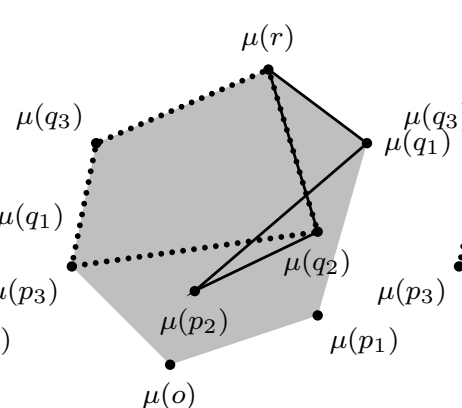

(e)

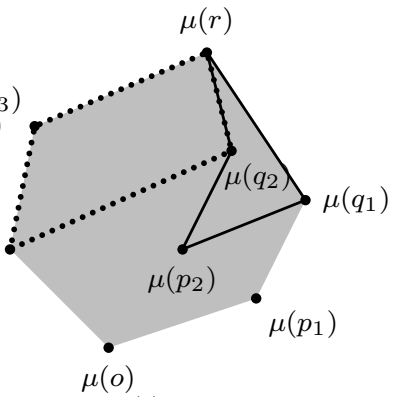

(f)

Figure 6.4. Possible configurations of $\Gamma$ of type Table 5.1.(h)

Case (ii): $\gamma_{p_{1}}$ contains $q_{1}$ and $q_{2}$. First, $\gamma_{p_{1}}$ cannot be crossed since $\left(p_{1}, q_{1}\right)$ and $\left(q_{1}, r\right)$ are boundary edges. Suppose that $\gamma_{p_{1}}$ is concave, see Figure 6.4.(b). Since $p_{1}, q_{1}$, and $r$ are boundary vertices of $\gamma_{p_{1}}$, $q_{2}$ should be contained in the interior of the convex hull $\operatorname{Conv}\left\{p_{1}, q_{1}, r\right\}$ by Lemma 5.10. Then $q_{2}$ cannot be adjacent to $p_{3}$ by Lemma 6.2. Also, $q_{2}$ cannot be adjacent to $o$ since $o$ is already adjacent to three vertices $p_{1}, p_{2}$, and $p_{3}$. Thus $q_{2}$ is adjacent to $p_{2}$ by the index increasing property. Then $p_{2}$ should be in the interior of $\square \gamma_{p_{1}}$ by Lemma 6.2 at $q_{2}$. Moreover, by Lemma 6.2 again, $p_{2}$ cannot be adjacent to $q_{3}$. Thus $p_{2}$ is adjacent to $q_{1}$ and this contradicts the three valency of $\Gamma$ at $p_{3}$. Therefore, $\gamma_{p_{1}}$ is convex.

Case (iii): $\gamma_{p_{2}}$ contains $q_{1}$ and $q_{2}$. Note that $p_{3}$ is adjacent to $q_{2}$ because $q_{1}$ is adjacent to $p_{1}$ and $p_{2}$, see Figure 6.4.(c). Suppose that $\gamma_{p_{2}}$ is crossed. Since the edge $\left(q_{1}, r\right)$ is boundary, two line segments $\overline{q_{2} r}$ and $\overline{p_{2} q_{1}}$ should intersect by Lemma 5.10. Then this contradicts to Lemma 5.12 with respect to the edge $\left(q_{2}, r\right)$, so $\gamma_{p_{2}}$ is not crossed, see Figure 6.4.(e). 
Next, suppose that $\gamma_{p_{2}}$ is concave. By the index increasing property, $p_{2}$ should be below $q_{1}$ and $q_{2}$. Thus $q_{2}$ should be lying on the interior of $\operatorname{Conv}\left\{p_{2}, q_{1}, r\right\}$, see Figure 6.4.(f). Then it contradicts Lemma 5.12 with respect to the edge $\left(q_{2}, r\right)$. Therefore, $\gamma_{p_{2}}$ is convex.

Case (iv): $\gamma_{p_{3}}$ contains $q_{1}$ and $q_{2}$. Such case does not happen by the three valency at $p_{3}$.

\section{REFERENCES}

[AB] M. F. Atiyah and R. Bott, The moment map and equivariant cohomology, Topology 23 (1984), No. 1, 1-28.

[BV] N. Berline and M. Vergne, Classes caractéristiques équivariantes. Formule de localisation en cohomologie équivariante, C. R. Acad. Sci. Paris 295 (1982), 539-541.

[At] M. F. Atiyah, Convexity and commuting Hamiltonians, Bull. London Math. Soc. 14 (1982), No. 1, 1-15.

$[\mathrm{Au}] \mathrm{M}$. Audin, Torus actions on symplectic manifolds, Second revised edition. Progress in Mathematics 93, Birkhäuser Verlag, Basel, 2004.

[Cho1] Y. Cho, Hard Lefschetz property of symplectic structures on compact Kähler manifolds, Trans. Amer. Math. Soc. 368 (2016), No. 11, 8223-8248.

[Cho2] Y. Cho, Unimodality of Betti numbers for Hamiltonian circle actions with index-increasing moment maps, Internat. J. Math. 27 (2016), No. 5, 1650043, 14pp.

[CK1] Y. Cho, M. K. Kim, Unimodality of the Betti numbers for Hamiltonian circle action with isolated fixed points, Math. Res. Lett. 21 (2014), No. 4, 691-696.

[CK2] Y. Cho, M. K. Kim, Hamiltonian circle action with self-indexing moment map, Math. Res. Lett. 23 (2016), 719-732.

[De] T. Delzant, Hamiltoniens périodiques et image convex de l'application moment, Bull. Soc. Math. France 116 (1988), 315-339.

[Fr] T. Frankel, Fixed points and torsion on Kahler manifolds, Ann. of Math. 70 (1959), 1-8.

[Go] R. Gompf, A new construction of symplectic manifolds, Ann. of Math. (2) 142 (1995), No. 3, 527-595.

[GKM] M. Goresky, R. Kottwitz, and R. MacPherson, Equivariant cohomology, Koszul duality, and the localization theorem, Invent. Math. 131 (1998), No. 1, 25-83.

[GS] V. Guillemin and S. Sternberg, Convexity properties of the moment mapping, Invent. Math. 67 (1982), No. 3, 491-513.

[GS2] V. Guillemin and S. Sternberg, Supersymmetry and equivariant de Rham theory, Springer-Verlag, 1999.

[GT] R. Goldin, S. Tolman, Towards Generalizing Schubert Calculus in the Symplectic Category, J. Symplectic Geom. 7 (2009), No. 4, 449-473.

[GZ] V. Guillemin, C. Zara, Combinatorial formulas for products of Thom classes, Geometry, mechanics, and dynamics, Springer, New York, 2002, 363-405.

[JHKLM] L. Jeffrey, T. Holm, Y. Karshon, E. Lerman, E. Meinrenken, Moment maps in various geometries, available online at http://www.birs.ca/workshops/2005/05w5072/report05w5072.pdf

[Ka] Y. Karshon, Periodic Hamiltonian flows on four-dimensional manifolds, Mem. Amer. Math. Soc. 141 (1999), No. 672.

[Ka2] Y. Karshon, Hamiltonian torus action, Geom. Phys. Lecture notes in Pure and Applied Mathematics Series 184, Marcel Dekker (1996), 221-230.

[Ki] F. C. Kirwan, Cohomology of quotients in symplectic and algebraic geometry, Princeton University Press, 1984.

[Km] M. K. Kim, Frankel's theorem in the symplectic category, Trans. Amer. Math. Soc. 358 (2006), No. 10, 4367-4377.

[Lu] S. Luo, The hard Lefschetz property for Hamiltlnian GKM manifolds, J. Algebr. Comb. 40, No. 1, 45-74.

[Ma] O. Mathieu, Harmonic cohomology classes of symplectic manifolds, Comment. Math. Helv. 70 (1995), No. 1, 1-9.

[Mc] P. McMullen, On simple polytopes, Invent. Math. 113 (1993), 419-444.

[Mo] D. Morton, GKM manifolds with low Betti numbers Ph.D. Thesis, University of Illinois at Urbana-Champaign, 2011.

[ST] S. Sabatini, S. Tolman, New techniques for obtaining Schubert-type formulas for Hamiltonian manifolds, J. Symplectic Geom. 11 (2013), No. 2, 179-230 .

[T] S. Tolman, Examples of non-Kähler Hamiltonian torus actions, Invent. Math. 131 (1998), No. 2, 299-310.

[We] M. J. Wenninger, Dual Models, Cambridge University Press, 1983.

[Wo] C. Woodward, Multiplicity-free Hamiltonian actions need not be Kähler, Invent. Math. 131 (1998), 311-319.

[Wo2] C. Woodward, Multiplicity-free Hamiltonian actions need not be Kähler, arXiv:dg-ga/9506009v1.

Department of Mathematics Education, Sungkyunkwan University, 25-2, Sungkyunkwan-ro, Jongno-gu, Seoul, 03063, Republic of Korea.

E-mail address: yunhyung@skku.edu

Department of Mathematics Education, Gyeongin National University of Education, 45 Gyodae-Gil, GyeyangGU, InCheon, 407-753, Republic of Korea

E-mail address: mkkim@kias.re.kr 